\title{
Liver Fibrosis and Metabolic Alterations in Adults With alpha- 1-antitrypsin Deficiency Caused by the Pi*ZZ Mutation
}

\author{
Karim Hamesch, ${ }^{1,2}$ Mattias Mandorfer, ${ }^{3}$ Vítor M. Pereira, ${ }^{4}$ Linda S. Moeller, ${ }^{5}$ \\ Monica Pons, ${ }^{6,7}$ Grace E. Dolman, ${ }^{8}$ Matthias C. Reichert, ${ }^{9}$ Carolin V. Schneider, ${ }^{2}$ \\ Vivien Woditsch, ${ }^{2}$ Jessica Voss, ${ }^{2}$ Cecilia Lindhauer, ${ }^{2}$ Malin Fromme ${ }^{2}$ Igor Spivak, ${ }^{2}$ \\ Nurdan Guldiken, ${ }^{2}$ Biaohuan Zhou, ${ }^{2}$ Anita Arslanow, ${ }^{9,10}$ Benedikt Schaefer, ${ }^{11}$ \\ Heinz Zoller, ${ }^{11}$ Elmar Aigner, ${ }^{12}$ Thomas Reiberger, ${ }^{3}$ Martin Wetzel, ${ }^{13}$ Britta Siegmund, ${ }^{13}$ \\ Carolina Simões, ${ }^{14}$ Rui Gaspar, ${ }^{15}$ Luís Maia, ${ }^{16}$ Dalila Costa, ${ }^{17}$ Mário Bento-Miranda, ${ }^{18}$ \\ Josef van Helden, ${ }^{19}$ Eray Yagmur, ${ }^{19}$ Danilo Bzdok, ${ }^{20,21}$ Jan Stolk, ${ }^{22}$ Wolfgang Gleiber, ${ }^{23}$ \\ Verena Knipel, ${ }^{24}$ Wolfram Windisch, ${ }^{24}$ Ravi Mahadeva, ${ }^{25}$ Robert Bals, ${ }^{26}$ \\ Rembert Koczulla, ${ }^{27,28}$ Miriam Barrecheguren, ${ }^{29}$ Marc Miravitlles, ${ }^{29}$ \\ Sabina Janciauskiene, ${ }^{30}$ Felix Stickel, ${ }^{31}$ Frank Lammert, ${ }^{9}$ Rodrigo Liberal, ${ }^{15}$ \\ Joan Genesca, ${ }^{6,7}$ William J. Griffiths, ${ }^{8}$ Michael Trauner, ${ }^{3}$ Aleksander Krag, ${ }^{5}$ \\ Christian Trautwein, ${ }^{1,2}$ and Pavel Strnad, ${ }^{1,2}$ on behalf of the \\ European Alpha1-Liver Study Group
}

\footnotetext{
${ }^{1}$ Coordinating Center for Alpha1-Antitrypsin Deficiency-Related Liver Disease of the European Reference Network "Rare Liver" and the European Association for the Study of the Liver Registry Group "Alpha1-Liver," University Hospital Aachen, Aachen, Germany; ${ }^{2}$ Medical Clinic III, Gastroenterology, Metabolic Diseases and Intensive Care, University Hospital Rheinisch-Westfälische Technische Hochschule Aachen, Aachen, Germany; ${ }^{3}$ Division of Gastroenterology and Hepatology, Department of Internal Medicine III, Medical University Vienna, Vienna, Austria; ${ }^{4}$ Department of Gastroenterology, Centro Hospitalar do Funchal, Madeira, Portugal; ${ }^{5}$ Department of Gastroenterology and Hepatology, Odense University Hospital, Odense, Denmark; ' ${ }^{6}$ Liver Unit, Hospital Universitari Vall d'Hebron, Vall d'Hebron Research Institute, Universitat Autonoma de Barcelona, Barcelona, Spain; ${ }^{7}$ Centro de Investigación Biomédica en Red de Enfermedades Hepáticas y Digestivas, Instituto de Salud Carlos III, Madrid, Spain; ${ }^{8}$ Department of Hepatology, Cambridge University Hospitals National Health Service Foundation Trust, Cambridge, UK; ${ }^{9}$ Department of Medicine II, Saarland University Medical Center, Saarland University, Homburg, Germany; ${ }^{10}$ Department of Internal Medicine I, University Medical Center of the Johannes Gutenberg-University, Mainz, Germany; ${ }^{11}$ Department of Internal Medicine I, Medical University Innsbruck, Innsbruck, Austria; ${ }^{12}$ Department of Internal Medicine I, Paracelsus Medical University, Salzburg, Austria; ${ }^{13}$ Department of Medicine I, Charité-Universitaetsmedizin Berlin, Campus Benjamin Franklin, Berlin, Germany; ${ }^{14}$ Gastroenterology Department, Centro Hospitalar Lisboa Norte, Lisbon, Portugal; ${ }^{15}$ Gastroenterology Department, Centro Hospitalar de São João, Faculty of Medicine of Porto University, Porto, Portugal; ${ }^{16}$ Gastroenterology Department, Centro Hospitalar do Porto, Porto, Portugal; ${ }^{17}$ Gastroenterology Department, Hospital de Braga, Braga, Portugal; ${ }^{18}$ Gastroenterology Department, Hospital Universitário de Coimbra, Coimbra, Portugal; ${ }^{19}$ Medical Care Centre, Dr Stein and Colleagues, Moenchengladbach, Germany; ${ }^{20}$ Department of Psychiatry, Psychotherapy and Psychosomatics, University Hospital Rheinisch-Westfälische Technische Hochschule Aachen, Aachen, Germany; ${ }^{21}$ Jülich Aachen Research Alliance-Brain, Aachen, Germany; ${ }^{22}$ Clinic for Pulmonology, Leiden University Medical Center, Leiden, The Netherlands; ${ }^{23}$ Clinic for Pulmonology, University Hospital Frankfurt, Frankfurt, Germany; ${ }^{24}$ Department of Pneumology, Cologne Merheim Hospital, Kliniken der Stadt Köln gGmbH, Witten/Herdecke University, Faculty of Health/ School of Medicine, Cologne, Germany; ${ }^{25}$ Department of Respiratory Medicine, Cambridge National Institute for Health Research, Biomedical Research Centre, University of Cambridge, Cambridge, UK; ${ }^{26}$ Department of Medicine V, Saarland University Medical Center, Saarland University, Homburg, Germany; ${ }^{27}$ Clinic for Pneumology, Marburg University Hospital, Marburg, Germany; ${ }^{28}$ Institute for Pulmonary Rehabilitation Research, Schoen Clinic Berchtesgadener Land, Member of the Deutsches Zentrum für Lungenforschung, Schönau am Königssee, Germany; ${ }^{29}$ Department of Pneumology, Vall d'Hebron University Hospital, Centro de Investigación Biomédica en Red de Enfermedades Respiratorias, Barcelona, Spain; ${ }^{30}$ Clinic for Pneumology, German Center for Lung Research, Medical University Hannover, Hannover, Germany; and ${ }^{31}$ Department of Gastroenterology and Hepatology, University Hospital of Zurich, Zurich, Switzerland
} 


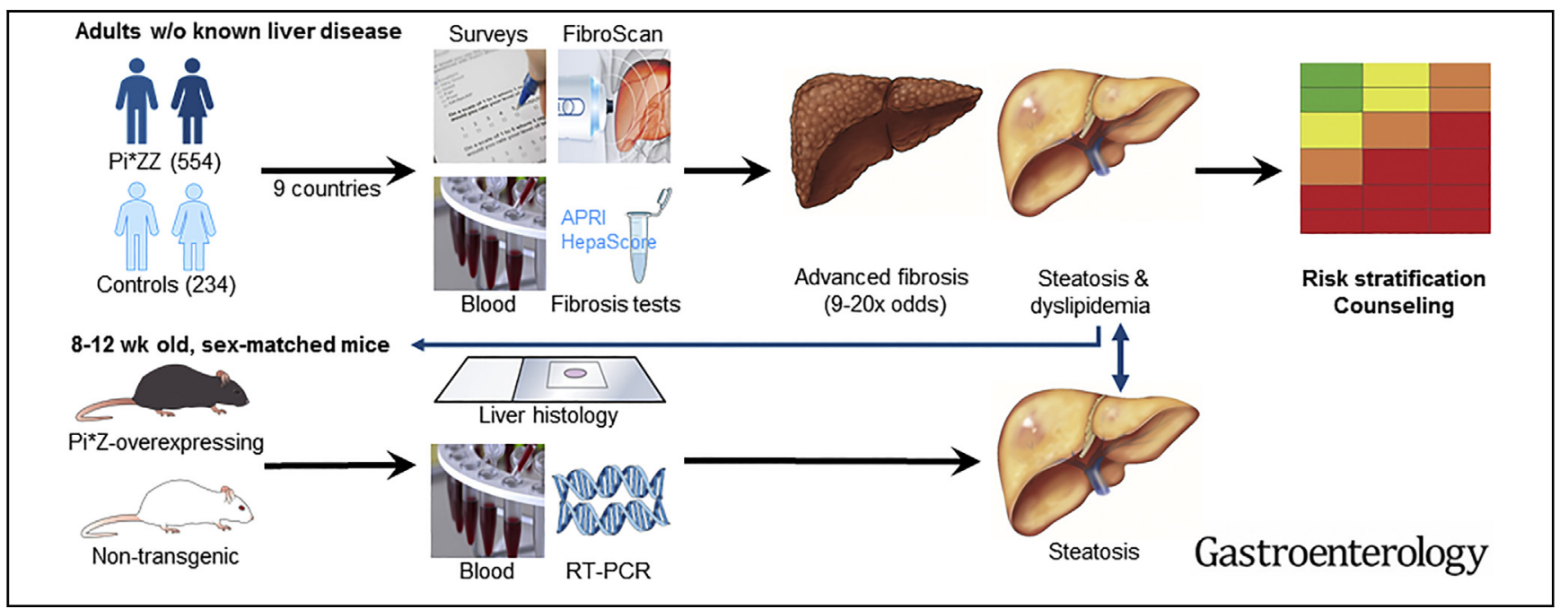

BACKGROUND \& AIMS: Alpha-1 antitrypsin deficiency (AATD) is among the most common genetic disorders. Severe AATD is caused by a homozygous mutation in the SERPINA1 gene that encodes the Glu342Lys substitution (called the Pi*Z mutation, Pi*ZZ genotype). Pi*ZZ carriers may develop lung and liver diseases. Mutation-associated lung disorders have been well studied, but less is known about the effects in liver. We assessed the liver disease burden and associated features in adults with this form of AATD. METHODS: We collected data from $554 \mathrm{Pi}^{*} \mathrm{ZZ}$ adults (403 in an exploratory cohort, 151 in a confirmatory cohort), in 9 European countries, with AATD who were homozygous for the $\mathrm{Pi} Z \mathrm{Z}$ mutation, and 234 adults without the $\mathrm{Pi}^{*} \mathrm{Z}$ mutation (controls), all without pre-existing liver disease. We collected data on demographic parameters, comorbidities, lung- and liver-related health, and blood samples for laboratory analysis. Liver fibrosis was assessed non-invasively via the serum tests Aspartate Aminotransferase to Platelet Ratio Index and HepaScore and via transient elastography. Liver steatosis was determined via transient elastography-based controlled attenuation parameter. We performed histologic analyses of livers from transgenic mice that overexpress the AATD-associated Pi*Z variant. RESULTS: Serum levels of liver enzymes were significantly higher in $\mathrm{Pi}^{*} \mathrm{ZZ}$ carriers vs controls. Based on non-invasive tests for liver fibrosis, significant fibrosis was suspected in $20 \%-36 \%$ of Pi*ZZ carriers, whereas signs of advanced fibrosis were 9- to 20-fold more common in $\mathrm{Pi}^{*} \mathrm{ZZ}$ carriers compared to non-carriers. Male sex; age older than 50 years; increased levels of alanine aminotransferase, aspartate aminotransferase, or $\gamma$-glutamyl transferase; and low numbers of platelets were associated with higher liver fibrosis burden. We did not find evidence for a relationship between lung function and liver fibrosis. Controlled attenuation parameter $\geq 280 \mathrm{~dB} / \mathrm{m}$, suggesting severe steatosis, was detected in $39 \%$ of $\mathrm{Pi}^{*} \mathrm{ZZ}$ carriers vs $31 \%$ of controls. Carriers of Pi*ZZ had lower serum concentrations of triglyceride and low- and very-lowdensity lipoprotein cholesterol than controls, suggesting impaired hepatic secretion of lipid. Livers from $\mathrm{Pi}^{*} \mathrm{Z}-$ overexpressing mice had steatosis and down-regulation of genes involved in lipid secretion. CONCLUSIONS: In studies of AATD adults with the Pi*ZZ mutation, and of $\mathrm{Pi}^{*} \mathrm{Z}$-overexpressing mice, we found evidence of liver steatosis and impaired lipid secretion. We identified factors associated with significant liver fibrosis in patients, which could facilitate hepatologic assessment and counseling of individuals who carry the Pi*ZZ mutation. ClinicalTrials.gov Number NCT02929940.

Keywords: TE; ALT; Rare Liver Disease; AST.

$\Lambda$ lpha-1 antitrypsin (AAT) is the major protease in$A$ hibitor ${ }^{1}$ and one of the most abundant glycoproteins in the serum. ${ }^{2}$ AAT is primarily responsible for inhibition of neutrophil elastase and proteinase 3 . Additionally, it has several immunomodulatory functions. ${ }^{3}$ AAT is the prototypic member of the serpin superfamily encoded by the SERPINA1 gene and is mainly produced by hepatocytes. ${ }^{4,5}$ After synthesis, AAT is translocated into the endoplasmic reticulum, where it is folded to be secreted from the hepatocyte into the bloodstream. 6,7 AAT variants, which are found in up to $10 \%$ of Caucasians, mostly interfere with the secretion process, leading to AAT accumulation in hepatocytes $^{3,8}$ and lack of AAT in the blood. ${ }^{1,5}$ The resulting condition, termed AAT deficiency (AATD), represents one of the most common genetic disorders leading to death. ${ }^{9}$ Severe AATD is caused mainly by the homozygous Pi*Z (Glu342Lys) mutation ( $\mathrm{Pi}^{*} \mathrm{ZZ}$ genotype, protease inhibitor [Pi]). $\mathrm{Pi}^{*} \mathrm{ZZ}$ carriers are highly susceptible to early-onset lung

Abbreviations used in this paper: AAT, alpha-1 antitrypsin; AATD, alpha-1 antitrypsin deficiency; ALT, alanine aminotransferase; APRI, Aspartate Aminotransferase to Platelet Ratio Index; AST, aspartate aminotransferase; CAP, controlled attenuation parameter; CAT, Chronic Obstructive Pulmonary Disease Assessment Test; $\mathrm{Cl}$, confidence interval; GGT, $\gamma$ glutamyl transferase; LSM, liver stiffness measurement; LTOT, long-term oxygen therapy; $\mathrm{OR}$, odds ratio; $\mathrm{Pi}$, protease inhibitor; $\mathrm{TE}$, transient elastography; ULN, upper limit of normal.

\section{Most current article}

\section{(C) 2019 by the AGA Institute} 0016-5085/\$36.00

https://doi.org/10.1053/j.gastro.2019.05.013 


\section{WHAT YOU NEED TO KNOW}

BACKGROUND

Alpha1-antitrypsin deficiency (AATD) can be caused by a homozygous mutation in the SERPINA1 gene that encodes the Glu342Lys substitution (called the $\mathrm{Pi}^{\star} Z$ mutation; $\mathrm{Pi}^{\star} \mathrm{ZZ}$ genotype). $\mathrm{Pi}^{\star} \mathrm{ZZ}$ carriers often develop lung and liver diseases; we assessed the liver disease burden and features in adults with this form of AATD.

\section{FINDINGS}

Alpha-1 antitrypsin deficiency (AATD) can be caused by a homozygous mutation in the SERPINA1 gene that encodes the Glu342Lys substitution (called the $\mathrm{Pi}^{\star} \mathrm{Z}$ mutation). $\mathrm{Pi}{ }^{\star} \mathrm{ZZ}$ carriers often develop lung and liver diseases; we assessed the liver disease burden and features in adults with this form of AATD.

\section{IMPACT}

This information could facilitate hepatologic assessment and counseling of patients who carry the $\mathrm{Pi}^{\star} \mathrm{ZZ}$ mutation.

\section{LIMITATIONS}

Most $\mathrm{Pi}^{\star} \mathrm{ZZ}$ carriers do not develop symptoms of AATD and remain undetected. These findings might not apply to this population.

emphysema and liver disease, representing the major causes of AATD-related mortality. ${ }^{4,5}$ Since the discovery of AATD more than 50 years ago, ${ }^{4,5}$ the Pi*ZZ-related lung disease has been a subject of intense research. It represents a loss-of-function phenotype, as deficiency of AAT is associated with accelerated destruction of lung parenchyma, leading to emphysema. ${ }^{4,5}$ This observation led to introduction of intravenous AAT augmentation as a treatment inhibiting the accelerated lung destruction and progression of emphysema. ${ }^{10,11}$

In contrast to $\mathrm{Pi}^{*} \mathrm{ZZ}$-related lung disease, there is a lack of knowledge for Pi*ZZ-related liver disease, which constitutes a toxic "gain-of-function" phenotype arising from hepatic accumulation of misfolded $\mathrm{Pi}^{*} \mathrm{Z}$ polymers. ${ }^{3,12}$ While lung emphysema usually develops in mid-life, $\mathrm{Pi}^{*}$ ZZ-related liver disease displays a biphasic pattern ${ }^{12,13}$; children surviving the critical first years of life have mostly unaltered serum liver enzymes during adolescence. ${ }^{14-16}$ The second peak of Pi*ZZ-related liver disease is thought to occur around 50 years of age, when a subset of Pi*ZZ carriers develop chronic liver disease, leading to progressive liver fibrosis. ${ }^{4,5,17}$ Nevertheless, the natural history as well as the prevalence of $\mathrm{Pi}^{*} \mathrm{ZZ}$-related liver disease in adults remain poorly characterized, ${ }^{18}$ and the current textbook knowledge is based mainly on small studies. This lack of published data is particularly striking because its prevalence among Caucasians $\left(1: 2000-4000^{4,14}\right)$ is higher than the prevalence of other, well-characterized liver disorders (eg, autoimmune hepatitis $1: 5900-9100,{ }^{19}$ primary sclerosing cholangitis $1: 6200-8000,{ }^{20}$ or Wilson disease $1: 30,000-100,000^{21}$ ). For example, a landmark study that revealed a high prevalence of cirrhosis in $\mathrm{Pi}^{*} \mathrm{ZZ}$ subjects relied on only 16 autopsied

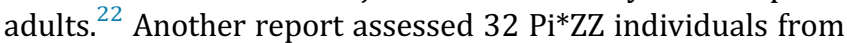
a Swedish birth screening program ${ }^{14}$ by acoustic radiation force impulse elastography. However, these patients were only 37-40 years old, that is, well before the age when clinically relevant $\mathrm{Pi}^{*} \mathrm{ZZ}$-related liver fibrosis is expected to occur. $^{23} \mathrm{~A}$ recent study evaluated the burden of liver fibrosis in a cohort of 94 biopsied Pi*ZZ carriers. ${ }^{24}$ Notably, it demonstrated a fair correlation between histologic liver fibrosis scores and non-invasive liver fibrosis assessments, thereby setting a rationale for evaluation of large, real-life patient cohorts.

Due to the shortage of knowledge, in particular with regard to non-invasive assessment of liver fibrosis burden, the current management of adult Pi*ZZ patients is based primarily on measurement of serum liver enzymes, although it has been described that they fail to reliably predict the course of disease. ${ }^{25}$ Led by the a priori hypothesis that $\mathrm{Pi}^{*} \mathrm{ZZ}$ carriers develop a liver disease more frequently than noncarriers, and that the $\mathrm{Pi}^{*} \mathrm{Z}$ retention within the endoplasmic reticulum results in multiple metabolic alterations, we performed, to our knowledge, the largest systematic assessment of liver disease burden and laboratory parameters in a multinational cohort of Pi*ZZ carriers. The goal of our study was to provide data needed for evidence-based patient management and counseling.

\section{Methods}

\section{Study Population}

A total of $554 \mathrm{Pi}^{*} \mathrm{ZZ}$ carriers and $234 \mathrm{Pi}^{*} \mathrm{Z}$ non-carriers (normal AAT phenotype) have been examined (Figure 1). Four hundred and three $\mathrm{Pi} * \mathrm{ZZ}$ and $151 \mathrm{Pi}^{*} \mathrm{ZZ}$ carriers comprised the exploratory (Table 1 and Supplementary Table 1) and confirmatory cohort (Supplementary Table 2), respectively. All participants were adults of self-reported European descent. Further details are given in the Supplementary Material (Recruitment of Study Population).

Pi*ZZ carriers from Germany, Austria, Switzerland, Great Britain, Denmark, Portugal, Spain, Belgium, and The Netherlands were recruited in collaboration with national patient advocacy groups, physicians with a specialist interest in AATD, as well as national AATD registries in these countries. Therefore, most carriers had a pre-existing diagnosis of AATD, either due to the presence of AATD-related lung disease or due to family screenings.

$\mathrm{Pi}^{*} \mathrm{Z}$ non-carriers had been recruited from genetically unrelated spouses of patients with known AATD, as well as volunteers during "liver awareness days," that are offered yearly by the University Hospital Aachen, Germany as a liver checkup for the general population. In all non-carriers, the serum level of AAT was determined and genotyping for the $\mathrm{Pi}^{*} \mathrm{Z}$ variant was performed. Individuals with AAT serum levels $\leq 110 \mathrm{mg} / \mathrm{dL}$ or presence of a $\mathrm{Pi}^{*} \mathrm{Z}$ variant were excluded from the study (10 individuals were excluded).

The inclusion criteria were a valid assessment via transient elastography (TE; FibroScan, Echosens, Paris, France) based on published recommendations ${ }^{26,27}$ and an ability to provide written informed consent. Main exclusion criteria were age younger than 18 years and pregnancy. All participants have been examined and all examinations (ie, questionnaires, clinical examination, blood sampling, and TE) were performed on the same day. A liver comorbidity was excluded by a 


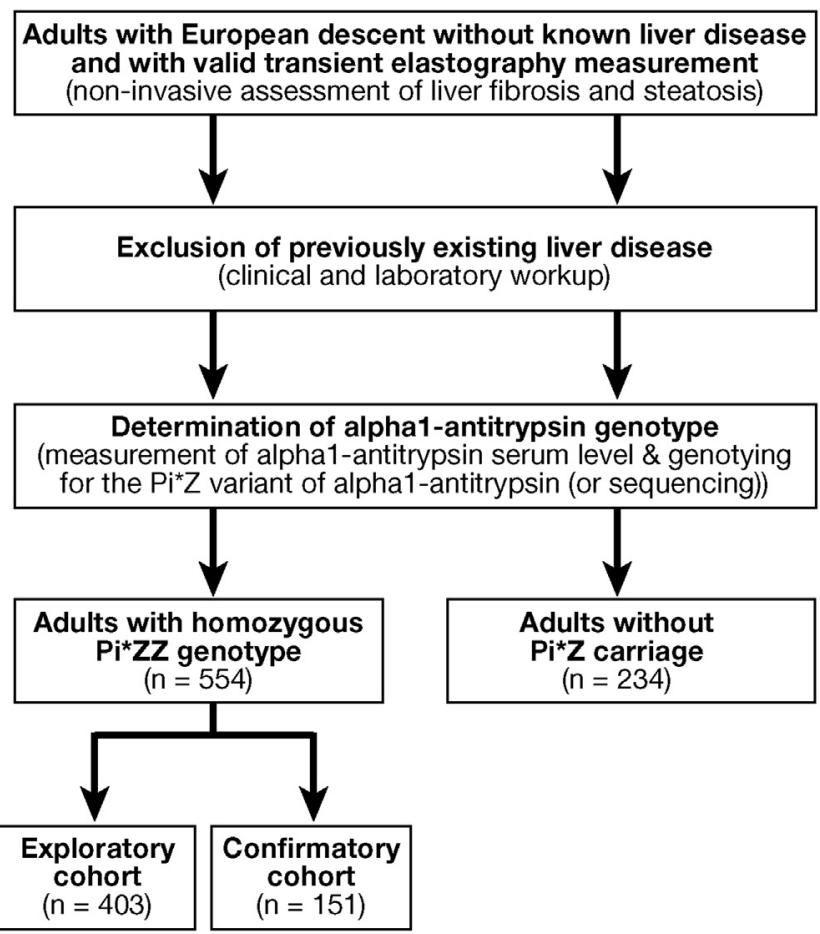

Figure 1. Overview of analyzed patients. $\mathrm{Pi}^{\star} \mathrm{Z}$ is the characteristic, disease-causing genetic variant of alpha- 1 antitrypsin. $\mathrm{Pi}^{\star} \mathrm{ZZ}$ is the homozygous carriage of the $\mathrm{Pi}^{\star} \mathrm{Z}$ variant.

comprehensive workup. TE constitutes the best-established method for non-invasive assessment of liver fibrosis via liver stiffness measurement (LSM) ${ }^{26,27}$ and is a reliable tool to assess steatosis via controlled attenuation parameter (CAP). ${ }^{28}$ Further details are given in the Supplementary Material (Assessment of Liver Disease and Ethical Statement).

\section{Assessment of Liver Disease}

In all participants, the presence of a previously diagnosed liver disease has been excluded by personal interview (ie, no established diagnosis of chronic liver disease and no history of liver resection or liver transplantation) and laboratory workup.

To non-invasively assess the degree of liver fibrosis, LSMs were performed by TE (FibroScan) using the M or XL probe according to published recommendations ${ }^{26}$ (eg, exclusion of confounders such as non-fasting or the presence of hepatic inflammation or cholestasis). At the same time, CAP was assessed as a surrogate of liver steatosis. Previously recommended, etiology-unspecific cutoffs for LSM and CAP were used. $^{26,28}$ For LSM, these cutoffs $(7.1 \mathrm{kPa}$, suggestive of significant fibrosis and $10.0 \mathrm{kPa}$, suggestive of advanced fibrosis) were chosen, as they imply a higher positive predictive value compared to the lower cutoffs described in the only study comparing LSM with histology ${ }^{24}(5.45 \mathrm{kPa}$ for significant fibrosis and $8.45 \mathrm{kPa}$ for advanced fibrosis; both with rather high negative predictive value, but low positive predictive value). TE measurements at all sites had been carried out by experienced physicians and only patients with at least 10 valid measurements and an interquartile range $\leq 30 \%$ of median LSM have been evaluated.

To further non-invasively assess the degree of liver fibrosis, we used 2 serum-based liver fibrosis tests. Aspartate
Aminotransferase (AST) to Platelet Ratio Index (APRI), an established liver fibrosis test comprising indirect markers, ${ }^{29}$ was calculated as follows: [AST (U/L) / 40] / platelet count (G/L). The HepaScore, a combination of direct and indirect liver fibrosis markers, ${ }^{30}$ was determined using the following parameters: age, sex, $\alpha 2$-macroglobulin, hyaluronic acid, bilirubin, and $\gamma$-glutamyl transferase (GGT). We employed previously suggested cutoffs for significant liver fibrosis (ie, F2 and higher) and advanced liver fibrosis (ie, F3 and higher) for both APRI and HepaScore. ${ }^{31-34}$

\section{Pi`Z-Overexpressing Mice and Quantification of Hepatic Steatosis}

Previously characterized transgenic, 8- to 12-week-old, sexmatched mice overexpressing the human $\mathrm{Pi}^{*} \mathrm{Z}$ variant ${ }^{35}$ and their non-transgenic littermates were analyzed. Further details are given in the Supplementary Material.

\section{Statistical Analysis}

Continuous variables were displayed as mean \pm SD and were compared by unpaired, 2-tailed $t$-tests, as well as by a multivariate linear model to account for confounders. Categorical variables were reported as absolute (n) and relative (\%) frequencies and contingency tables were analyzed with $\chi^{2}$ tests. Differences between groups were assessed by univariate and forward-stepwise multiple logistic regression analyses to calculate odds ratios (OR). ORs were given with their corresponding 95\% confidence intervals (CIs). Multivariable logistic regression was used to test for independent associations. Linear correlations between clinical variables and serum biomarkers or elastography parameters were assessed by Pearson or Spearman correlation coefficients, where appropriate.

Nominal $P$ values were reported for all statistical tests. The Bonferroni correction accounted for multiple testing. Differences were considered to be statistically significant when $P<$.05. Statistical analyses were performed using SPSS, version 23 (IBM, Armonk, NY) and graphs were created with Prism 5 (GraphPad, La Jolla, CA).

\section{Results}

\section{Clinical and Biochemical Characteristics of $P i^{*} Z Z$ Carriers}

First, we assessed 403 adult Pi*ZZ carriers and 234 adult non-carriers, both without previously known liver disease and without clinical or laboratory signs of previously existing liver disease (exploratory cohort; Figure 1). Both groups had similar age, sex distribution, body mass index values, and comparable rates of diabetes mellitus (Table 1). Relevant alcohol consumption ( $>12 \mathrm{~g} / \mathrm{d}$ for women and $>24 \mathrm{~g} / \mathrm{d}$ for men) was infrequent in both groups (Table 1). As expected, $\mathrm{Pi}^{*} \mathrm{ZZ}$ carriers had significantly higher Chronic Obstructive Pulmonary Disease Assessment Test (CAT) scores (a questionnaire measuring the health-related quality of life) and $26 \%$ required long-term oxygen therapy (LTOT) as an indicator of severe AATD-related lung disease (Table 1). Interestingly, while $90 \%$ of $\mathrm{Pi}^{*} \mathrm{ZZ}$ carriers underwent regular lung checkups, only $45 \%$ were subjected to measurements of serum liver enzymes at least once a 
Table 1. Characteristics of Homozygous Carriers of the alpha-1 antitrypsin $\mathrm{Pi}^{\star} \mathrm{Z}$ Variant $\left(\mathrm{Pi}^{\star} \mathrm{ZZ}\right)$ and $\mathrm{Pi}^{\star} \mathrm{Z}$ Non-Carriers (Exploratory Cohort)

\begin{tabular}{|c|c|c|c|}
\hline Variable & Non-carriers $(n=234)$ & Carriers $\left(\mathrm{Pi}^{\star} \mathrm{ZZ}\right)(\mathrm{n}=403)$ & $P$ value \\
\hline \multicolumn{4}{|l|}{ Characteristics } \\
\hline Age, $y$ & $53.1 \pm 14.6$ & $54.1 \pm 13.0$ & .39 \\
\hline Women & 48.7 & $4 \overline{5.4}$ & .42 \\
\hline $\mathrm{BMI}, \mathrm{kg} / \mathrm{m}^{2}$ & $25.1 \pm 3.8$ & $24.8 \pm 4.4$ & .34 \\
\hline Mean alcohol consumption, $g / d$ & $7.9 \pm 10.1$ & $6.6 \pm 10.3$ & .14 \\
\hline AAT serum level, ${ }^{a} m g / d L^{b}$ & $139.7 \pm 25.3$ & $28.6 \pm 16.6$ & $<.001$ \\
\hline \multicolumn{4}{|l|}{ Risk factors } \\
\hline $\mathrm{BMI} \geq 30 \mathrm{~kg} / \mathrm{m}^{2}$ & 14.9 & 13.0 & .51 \\
\hline Waist circumference, $\mathrm{cm}$ & $102.0 \pm 4.6$ & $95.0 \pm 14.2$ & .40 \\
\hline Diabetes mellitus & $5 . \overline{6}$ & 5.0 & .78 \\
\hline Relevant alcohol intake ${ }^{c}$ & 12.8 & 10.4 & .38 \\
\hline \multicolumn{4}{|l|}{ Lung status } \\
\hline Cigarette consumption, pack-years & $9.3 \pm 17.8$ & $10.0 \pm 14.0$ & .62 \\
\hline CAT score (points) ${ }^{b}$ & $6.8 \pm 6.0$ & $16.9 \pm 7.7$ & $<.001$ \\
\hline Long-term oxygen treatment $^{b}$ & 0.4 & 25.8 & $<.001$ \\
\hline \multicolumn{4}{|l|}{ Liver status } \\
\hline Liver stiffness, $k P a^{b}$ & $4.6 \pm 1.7$ & $6.7 \pm 5.8$ & $<.001$ \\
\hline Liver stiffness $\geq 7.1 \mathrm{kPa}^{b}$ & $6 . \overline{4}$ & 23.6 & $<.001$ \\
\hline Liver stiffness $\geq 10.0 \mathrm{kPa}^{b, d}$ & 1.3 & 13.6 & $<.001$ \\
\hline $\mathrm{CAP}, d B / m^{b}$ & $246 \pm 59$ & $267 \pm 57$ & $<.001$ \\
\hline $\mathrm{CAP} \geq 248 \mathrm{~dB} / \mathrm{m}^{e}$ & 48.2 & 61.1 & .002 \\
\hline $\mathrm{CAP} \geq 280 \mathrm{~dB} / \mathrm{m}^{f, g}$ & 28.4 & 38.7 & .012 \\
\hline APRI, units ${ }^{b}$ & $0.27 \pm 0.12$ & $0.41 \pm 0.36$ & $<.001$ \\
\hline APRI $\geq 0.50$ units $^{e}$ & 5.4 & 19.6 & $<.001$ \\
\hline $\mathrm{APRI} \geq 1.00$ units $^{e, h}$ & 0.5 & 4.5 & .005 \\
\hline HepaScore, units $^{b}$ & $0.25 \pm 0.21$ & $0.43 \pm 0.32$ & $<.001$ \\
\hline HepaScore $\geq 0.48$ units $^{b}$ & 13.5 & 36.3 & $<.001$ \\
\hline HepaScore $\geq 0.72$ units $^{b, i}$ & 4.1 & 25.6 & $<.001$ \\
\hline
\end{tabular}

NOTE. Quantitative measures are expressed as mean \pm SD or as relative frequency (\%). The cutoffs used for the non-invasive liver parameters are according to etiology-unspecific recommendations from the literature: liver stiffness $\geq 7.1 \mathrm{kPa}, \mathrm{APRI}$ $\geq 0.50$ units, and HepaScore $\geq 0.48$ units as indicators of significant fibrosis (fibrosis stage $\geq 2$ ) and liver stiffness $\geq 10.0 \mathrm{kPa}$, APRI $\geq 1.00$ units, and HepaScore $\geq 0.72$ units as surrogates of advanced fibrosis (fibrosis stage $\geq 3$ ).

BMI, body mass index.

${ }^{a}$ AAT serum levels of $\mathrm{Pi}^{\star} \mathrm{Z}$ non-carriers and $\mathrm{Pi}^{\star} \mathrm{ZZ}$ subjects, who did not receive AAT augmentation therapy, are shown. Mean AAT serum level of all $\mathrm{Pi}^{\star} \mathrm{ZZ}$ patients was $72.5 \pm 52.6 \mathrm{mg} / \mathrm{dL}$.

${ }^{b} P<.001$ (both univariable and multivariable analysis).

${ }^{c}$ Alcohol intake $>12 \mathrm{~g} / \mathrm{d}$ for women and $>24 \mathrm{~g} / \mathrm{d}$ for men (individuals with alcohol consumption $>40 \mathrm{~g} / \mathrm{d}$ for females or $>60$ $\mathrm{g} / \mathrm{d}$ for males had been excluded a priori).

${ }^{2}$ Adjusted OR, 19.8; 95\% Cl, 4.6-84.1.

${ }^{e} P<.01$ (both univariable and multivariable analysis).

${ }^{f}$ Adjusted OR, 2.1; 95\% Cl, 1.3-3.2.

${ }^{g} P<.02$ (both univariable and multivariable analysis).

${ }^{h}$ Adjusted OR, 9.5; 95\% Cl, 1.2-76.6.

'Adjusted OR, 14.8; 95\% Cl, 5.4-40.4. All multivariable analyses were adjusted for age, sex, BMI, presence of diabetes mellitus, and mean alcohol consumption.

year and only $21 \%$ received regular liver ultrasound examinations.

Serum liver enzymes were increased above the sexspecific upper limit of normal (ULN) in only a minority of $\mathrm{Pi}^{*} \mathrm{ZZ}$ carriers: $19.1 \%$ alanine aminotransferase (ALT), $12.7 \%$ AST, $23.7 \%$ GGT, and $8.6 \%$ alkaline phosphatase (Supplementary Table 3). The mean serum ALT, AST, and GGT activities were higher in $\mathrm{Pi}^{*} \mathrm{ZZ}$ carriers than non-carriers $(80 \%$ vs $66 \%$ of ULN, $74 \%$ vs $62 \%$ of ULN, $100 \%$ vs $58 \%$ of ULN; all, $P<.001$ ) (Figure $2 A-C$ ). The differences in cholestatic markers were less obvious (alkaline phosphatase: $66 \%$ vs $60 \%$ of ULN, $P=.003$; bilirubin: $51 \%$ vs $47 \%$ of ULN, $P=.11$ ) (Figure $2 D-E$ ). Surrogate markers of advanced liver disease, such as platelet count (marker of portal hypertension), as well as albumin and international normalized ratio (markers of hepatic synthesis) were within the normal range in most patients (Supplementary Table 3). Nevertheless, Pi*ZZ carriers displayed lower platelet count and albumin, but higher international normalized ratio values than non-carriers (all, $P<.001$ ) (Figure $2 F$; Supplementary Table 3). Notably, Pi*ZZ carriers also had higher hemoglobin levels, that might be due to a higher burden of lung disease (Supplementary Table 3). All of the parameters mentioned remained significant in a multivariable analysis adjusting for age, sex, body mass index, presence of diabetes mellitus, and mean alcohol consumption (all, $P<.05$ ) (Supplementary Table 3). 

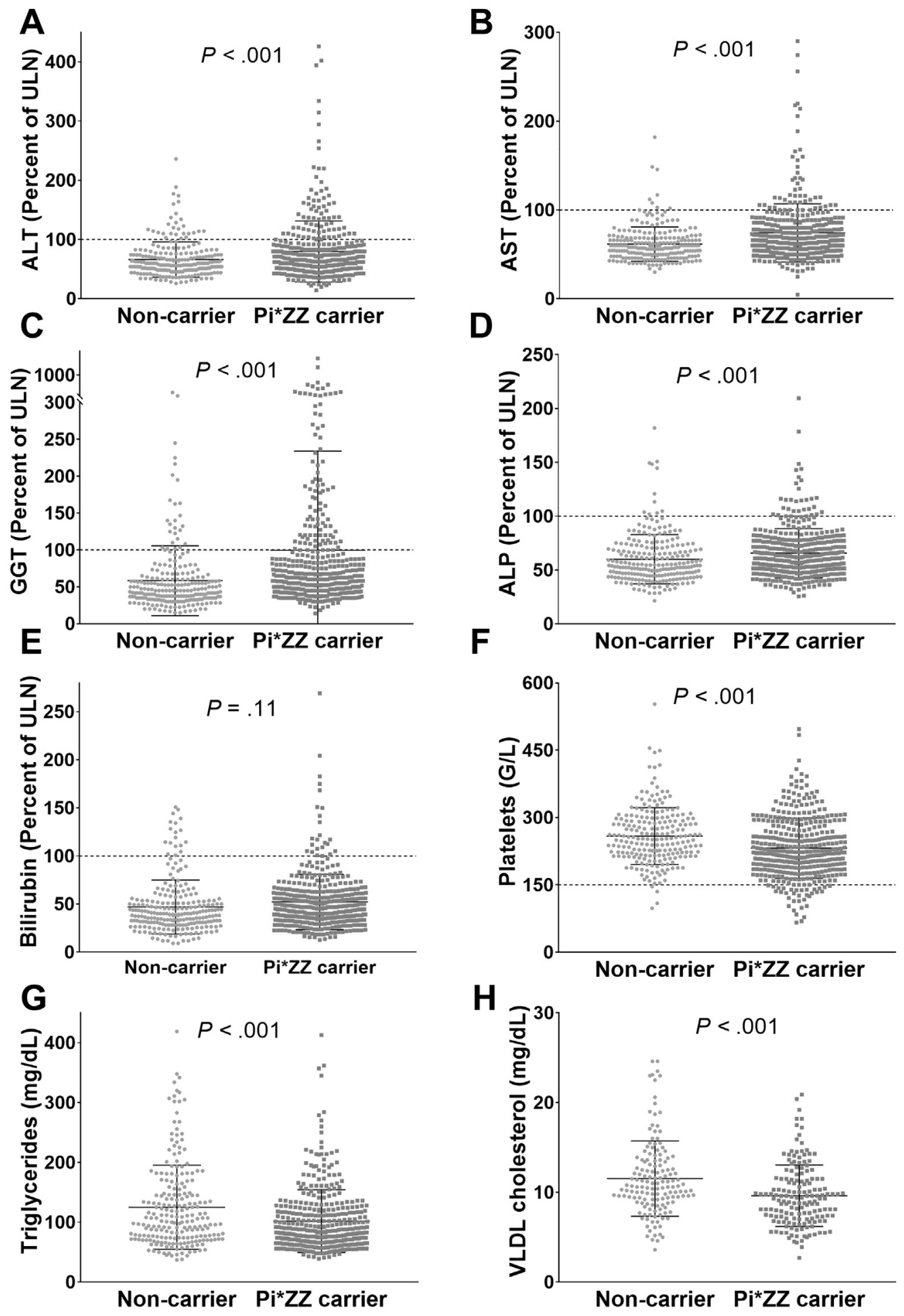

Figure 2. Liver-related and metabolic parameters in carriers homozygous for the AAT $\mathrm{Pi}^{\star} \mathrm{Z}$ variant $\left(\mathrm{Pi}^{\star} \mathrm{ZZ}\right)$ and $\mathrm{Pi}^{\star} \mathrm{Z}$ noncarriers. After fasting, 403 $\mathrm{Pi}^{\star} \mathrm{ZZ}$ carriers and 234 non-carriers without known liver disease were subjected to laboratory analysis. $(A-E)$ Scatter plots depict liver enzyme serum activities normal-

H

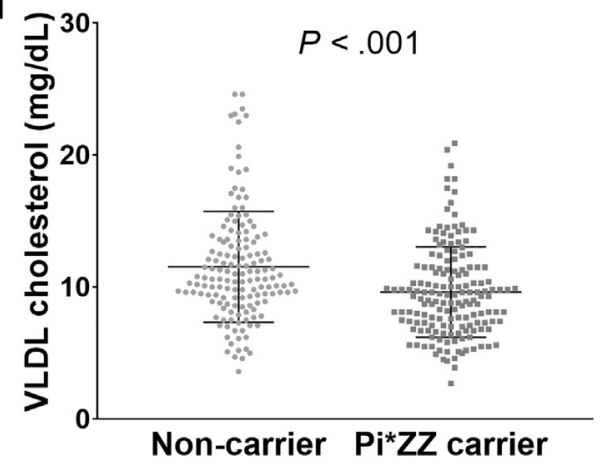
ized to the sex-specific ULN that is marked as a dotted line. $(F)$ Scatter plots of platelet count in the blood (lower limit of normal is marked as a dotted line). (G-H) Scatter plots of absolute serum levels of triglycerides and VLDL cholesterol. ALP, alkaline phosphatase; VLDL, very low-density lipoprotein.

Taken together, serum liver enzymes were significantly higher in $\mathrm{Pi}^{*} \mathrm{ZZ}$ carriers compared to non-carriers, but only a minority of Pi*ZZ carriers had values above the ULN.

\section{Non-Invasive Liver Fibrosis Tests in Pi*ZZ Carriers}

TE revealed that the mean LSMs were higher in $\mathrm{Pi}^{*} \mathrm{ZZ}$ carriers compared to non-carriers $(6.7 \pm 5.8 \mathrm{kPa}$ vs $4.6 \pm 1.7$
$\mathrm{kPa}, P<.001$ ) (Table 1 and Figure $3 A$ ). In line, 23.6\% of $\mathrm{Pi}^{*} \mathrm{ZZ}$ carriers vs $6.4 \%$ of non-carriers displayed LSM $\geq 7.1$ $\mathrm{kPa}$, suggesting the presence of significant liver fibrosis (fibrosis stage of at least 2) $(P<.001)$ (Table 1). The difference between carriers and non-carriers was even more pronounced when considering subjects with $\mathrm{LSM} \geq 10.0 \mathrm{kPa}$, suggesting the presence of advanced fibrosis stage of at least 3 (13.6\% vs $1.3 \%$; adjusted OR, 19.8; 95\% CI, 4.6-84.1). 
A

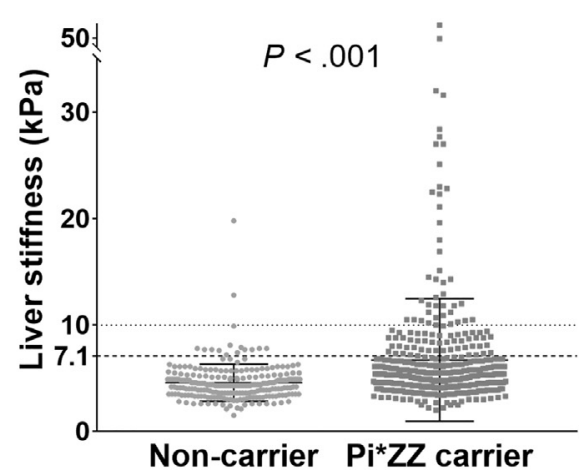

C

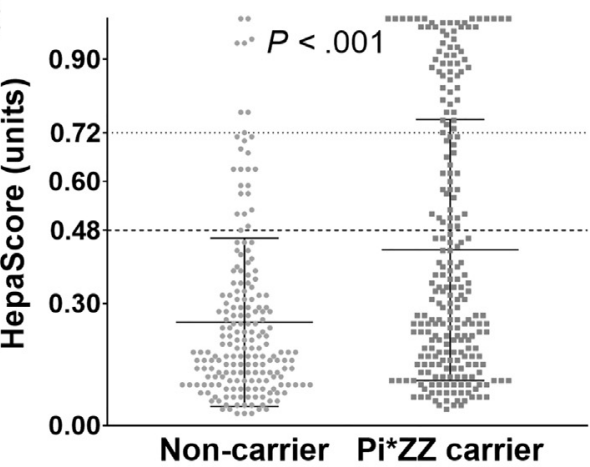

B

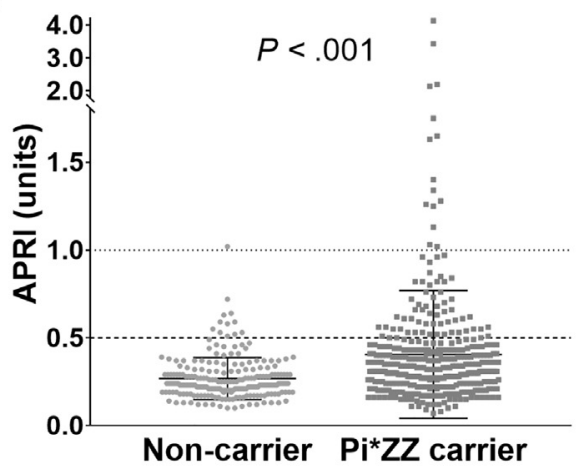

D

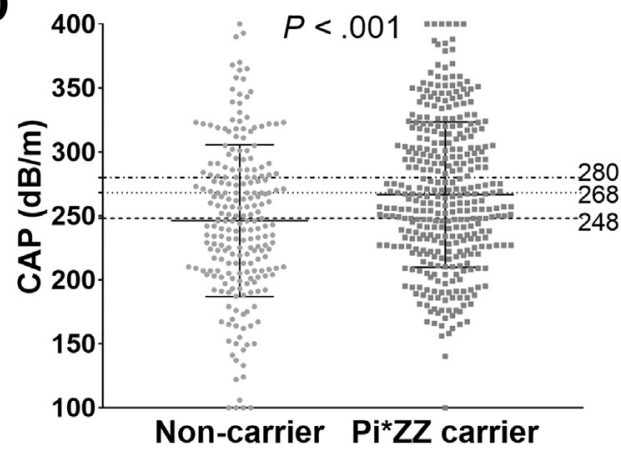

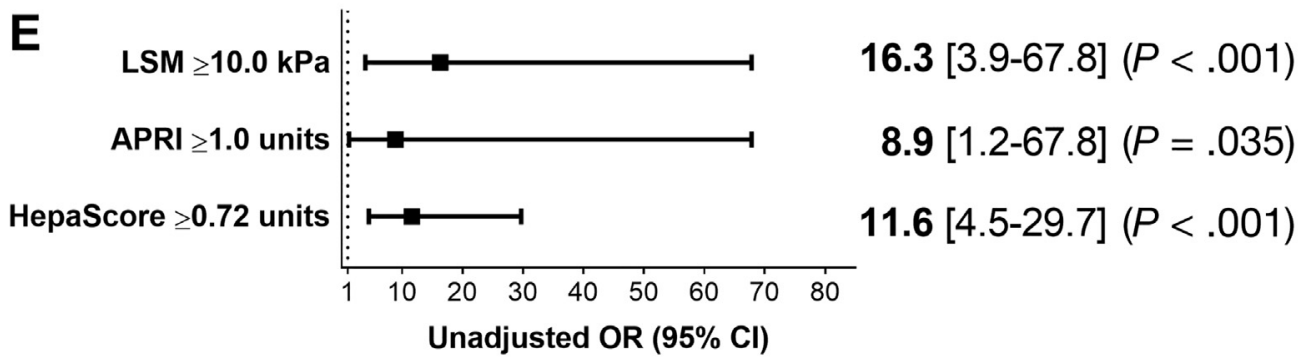

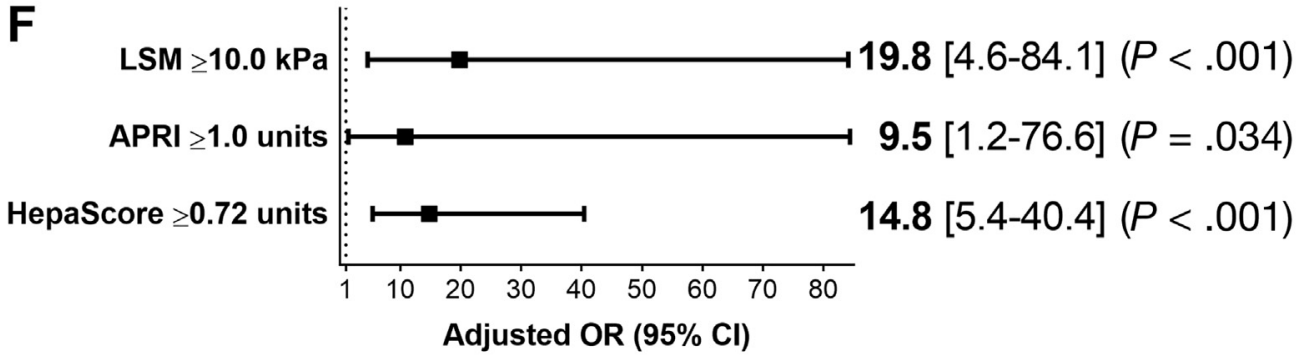

Figure 3. Non-invasive liver fibrosis and steatosis parameters in carriers homozygous for the AAT $\mathrm{Pi}^{\star} \mathrm{Z}$ variant $\left(\mathrm{Pi}^{\star} \mathrm{ZZ}\right)$ and $\mathrm{Pi}^{\star} \mathrm{Z}$ non-carriers. After fasting, $403 \mathrm{Pi} Z Z Z$ carriers and 234 non-carriers without known liver disease (exploratory cohort) were subjected to laboratory analysis and non-invasive assessment by TE (FibroScan). (A) Scatter plot of median liver stiffness determined by TE. The dotted lines indicate the following etiology-unspecific cutoff levels: $7.1 \mathrm{kPa}$ (suggestive of fibrosis stage $\geq 2$ ) and $10.0 \mathrm{kPa}$ (suggestive of fibrosis stage $\geq 3$ ). (B) Scatter plots of APRI values, an indirect fibrosis marker in the blood. The dotted lines indicate the following etiology-unspecific cutoff levels: 0.5 (suggestive of fibrosis stage $\geq 2$ ) and 1.0 (suggestive of fibrosis stage $\geq 3$ ). (C) Scatter plots of HepaScore values, a direct fibrosis serum marker. The dotted lines indicate the following etiology-unspecific cutoff levels: 0.48 (suggestive of fibrosis stage $\geq 2$ ) and 0.72 (suggestive of fibrosis stage $\geq 3$ ). (D) Scatter plot of CAP, a surrogate parameter of liver steatosis determined by TE. The dotted lines indicate the following etiologyunspecific cutoff levels: $248 \mathrm{~dB} / \mathrm{m}$ (suggestive of steatosis stage $\geq 1$ ), $268 \mathrm{~dB} / \mathrm{m}$ (suggestive of steatosis stage $\geq 2$ ), and $280 \mathrm{~dB} / \mathrm{m}$ (suggestive of steatosis stage 3). Univariable $(E)$ and multivariable $(F)$ analyses depicts the odds of $\mathrm{Pi}^{\star} Z Z$ carriers (compared to non-carriers) to display values suggestive of advanced fibrosis. The adjustments were made for sex, age, body mass index, presence of diabetes mellitus, and mean alcohol consumption. 
In agreement with LSM, APRI-a serum-based, indirect liver fibrosis test-showed higher levels in Pi*ZZ carriers vs non-carriers $(0.41 \pm 0.36$ units vs $0.27 \pm 0.12$ units; $P<.001$ ) (Table 1 and Figure $3 B$ ). Comparable APRI and LSM values were seen in the national subcohorts (Supplementary Table 1). Of $\mathrm{Pi}^{*} \mathrm{ZZ}$ carriers, $19.6 \%$ had APRI values $\geq 0.50$ units, suggesting the presence of significant liver fibrosis, compared to $5.4 \%$ of non-carriers $(P<.001)$ (Table 1 ). Similarly, $4.5 \%$ of $\mathrm{Pi}^{*} \mathrm{ZZ}$ carriers vs $0.5 \%$ of non-carriers displayed APRI $\geq 1.0$ units, suggesting the presence of advanced liver fibrosis (adjusted OR, 9.5; 95\% CI, 1.2-76.6). The HepaScore, a serum-based, direct liver fibrosis test, revealed higher levels in $\mathrm{Pi}^{*} \mathrm{ZZ}$ carriers compared to noncarriers $(0.43$ units \pm 0.32 vs $0.25 \pm 0.21$ units; $P<.001$ ) (Table 1 and Figure $3 C$ ). HepaScore values suggestive of significant and advanced liver fibrosis were also more common in $\mathrm{Pi}^{*} \mathrm{ZZ}$ carriers vs non-carriers (significant fibrosis [HepaScore $\geq 0.48$ units]: $36.3 \%$ vs $13.5 \%$; $P<.001$ and advanced fibrosis (HepaScore $\geq 0.72$ units): $25.6 \%$ vs $4.1 \%$; $P<.001$; adjusted OR, 14.8; 95\% CI, 5.4-40.4, respectively) (Table 1). The 3 liver fibrosis tests correlated reasonably well with each other (LSM and APRI: $\rho=.46, P<.001$; LSM and HepaScore: $\rho=.38, P<.001$; APRI and HepaScore: $\rho=.43$, $P<.001$ ) (Supplementary Figure 1) and collectively allowed a convenient triage of $\mathrm{Pi}^{*} \mathrm{ZZ}$ carriers. For example, based on APRI and LSM, $68.1 \%$ of Pi*ZZ carriers were unlikely to have significant liver fibrosis (ie, were classified as no or minimal fibrosis [F0-1] by both scores). On the other hand, $10.9 \%$ and $3.1 \%$ had values consistent with significant, advanced fibrosis, based on both APRI and LSM, respectively.

While our study cannot offer a precise calculation of liver fibrosis burden, it suggests that $20 \%-36 \%$ of $\mathrm{Pi}^{*} \mathrm{ZZ}$ carriers had significant liver fibrosis and that the odds for having advanced liver fibrosis were 9-20 times higher in $\mathrm{Pi}^{*} \mathrm{ZZ}$ carriers vs non-carriers (Figure $3 E$ and $F$ ).

\section{Confirmatory Pi`ZZ Cohort}

To further corroborate our findings, we took advantage of an independent Pi*ZZ cohort that was recruited in the same manner as the exploratory cohort. This confirmatory cohort consisted of $151 \mathrm{Pi}^{*} \mathrm{ZZ}$ patients from 4 European countries, and all national subcohorts had a similar demographic pattern (Supplementary Table 2). Moreover, there were no major differences in the demographic characteristics and the fibrosis test measures between the confirmatory and the exploratory cohorts (Supplementary Tables 1 and 2). Because the majority of patients in our study were recruited in Germany, we pooled the exploratory and confirmatory cohorts to compare German and nonGerman recruits. While German Pi*ZZ carriers were somewhat older and had a stronger AATD-related lung disease than non-German recruits, no other relevant differences between groups were detected (Supplementary Table 4).

\section{Non-Invasive Steatosis Assessment in $\mathrm{Pi}^{\star} \mathrm{ZZ}$ Carriers and Steatosis Assessment in $\mathrm{Pi}^{*} \mathrm{Z}$ - Overexpressing Mice}

CAP, an established surrogate of liver steatosis, ${ }^{28}$ revealed higher mean values in $\mathrm{Pi}^{*} \mathrm{ZZ}$ carriers vs non-carriers
$(267 \pm 57 \mathrm{~dB} / \mathrm{m}$ vs $246 \pm 56 \mathrm{~dB} / \mathrm{m} ; P<.001)$ (Table 1$)$. Accordingly, more $\mathrm{Pi}^{*} \mathrm{ZZ}$ carriers than non-carriers displayed CAP, suggesting the presence of mild steatosis (CAP $\geq 248 \mathrm{~dB} / \mathrm{m}: 61.1 \%$ vs $48.2 \%$, respectively; $P=.002$ ) and severe steatosis (CAP $\geq 280 \mathrm{~dB} / \mathrm{m}: 38.8 \%$ vs $28.2 \%$, respectively; $P=.012$; adjusted $\mathrm{OR}, 2.1 ; 95 \% \mathrm{CI}, 1.4-3.3$ ) (Table 1 and Figure $3 D$ ). All results remained significant in a multivariable analysis (all, $P<.01$ ) (Table 1).

To investigate whether these differences in CAP values constitute a direct consequence of $\mathrm{Pi}^{*} \mathrm{Z}$ retention in the liver, we examined transgenic mice overexpressing the human $\mathrm{Pi}^{*} \mathrm{Z}$ variant and their non-transgenic littermates. On histologic sections, Pi*Z-overexpressing mice showed more steatosis than their non-transgenic littermates (Figure 4A). To quantify the extent of steatosis, we performed Oil Red 0 staining and assessment of hepatic triglyceride content. Both analyses revealed a stronger lipid accumulation in $\mathrm{Pi}^{*} \mathrm{Z}$-overexpressing mice compared to non-transgenic littermates (Figure $4 B$ and $C$ ). In contrast, no difference in serum cholesterol or serum triglyceride levels was observed (Figure $4 D$ and $E$ ). Because Pi*Z-overexpressing mice were shown to have decreased levels of hepatocyte nuclear factor $4 \alpha$, which is essential for maintenance of liver homeostasis, ${ }^{36,37}$ we analyzed its expression as well as the expression of its downstream targets involved in lipid secretion. Notably, hepatocyte nuclear factor $4 \alpha$, microsomal triglyceride transfer protein, apolipoprotein A4, and apolipoprotein C2 were all significantly down-regulated in $\mathrm{Pi}^{*}$ Z-overexpressing mice compared to their non-transgenic littermates (Figure $4 F$ ).

The differences in hepatic steatosis in both humans and mice prompted us to evaluate serum lipid parameters. $\mathrm{Pi}^{*} \mathrm{ZZ}$ carriers had, compared to non-carriers, markedly reduced serum triglyceride $(102 \pm 52 \mathrm{mg} / \mathrm{dL}$ vs $125 \pm 70 \mathrm{mg} / \mathrm{dL}$; $P<.001)$ and very-low-density lipoprotein cholesterol concentrations $(9.6 \pm 3.4 \mathrm{mg} / \mathrm{dL}$ vs $11.5 \pm 4.2 \mathrm{mg} / \mathrm{dL}$; $P<.001$ ). Only a minimal, but a significant, difference in low-density lipoprotein cholesterol $(P=.03)$ and no significant alterations in total cholesterol were found (Figure $2 G$ and $H$ and Supplementary Table 3). Multivariable adjustment confirmed these differences (all, $P<.001$ ) (Supplementary Table 3). While 58\% of Pi*ZZ carriers received AAT augmentation therapy, the observed findings regarding liver fibrosis and steatosis, as well as changes in lipid parameters, were also detected when only Pi*ZZ individuals without augmentation therapy were considered (Supplementary Table 5).

\section{Impact of Age, Lung Involvement, and Sex on Pi*ZZ-Related Liver Alterations}

The availability of a large, real-life cohort of $\mathrm{Pi}^{*} \mathrm{ZZ}$ carriers allowed us to assess whether age, sex, and presence of lung disease have any association with the extent of liver fibrosis. As expected, lung-related parameters revealed an age-dependent deterioration of pulmonary function that was evident in higher CAT values, higher rates of LTOT, and subsequently higher need for AAT augmentation therapy (Supplementary Table 6). In 
A
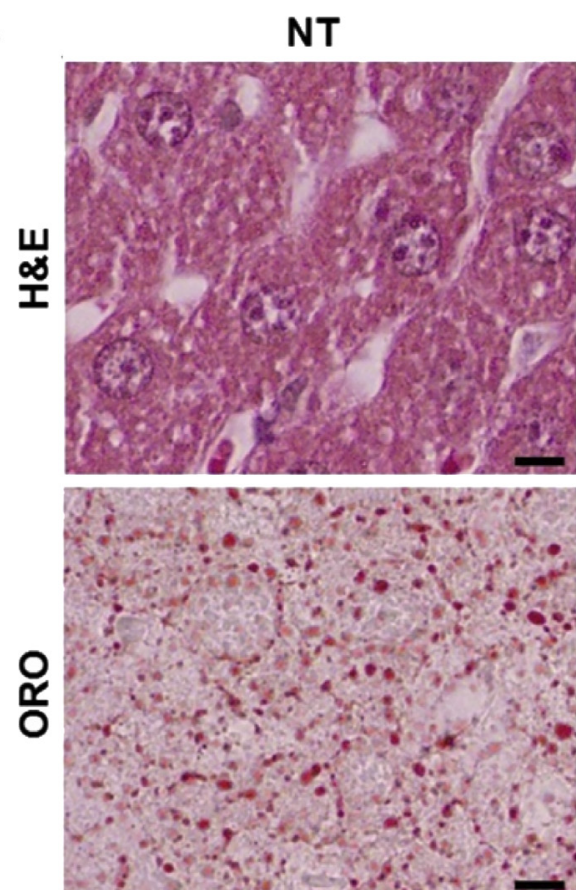

PiZ
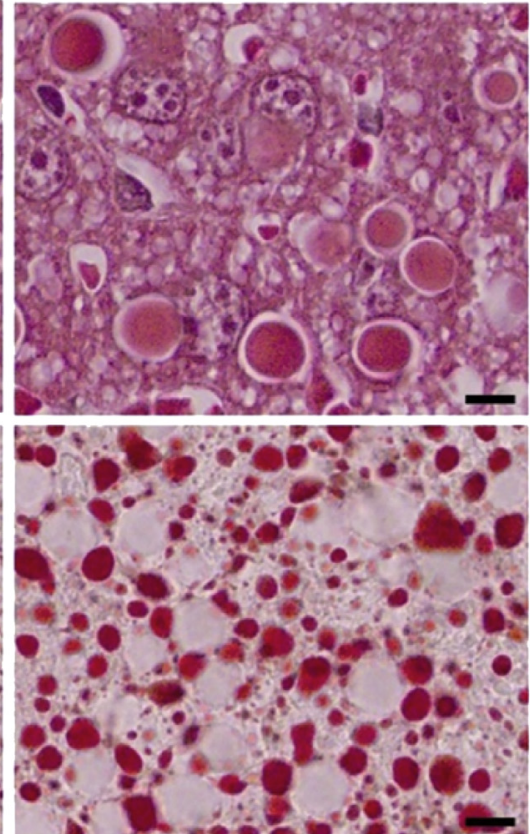

B

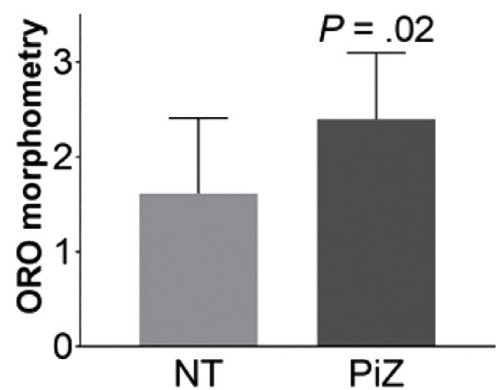

C

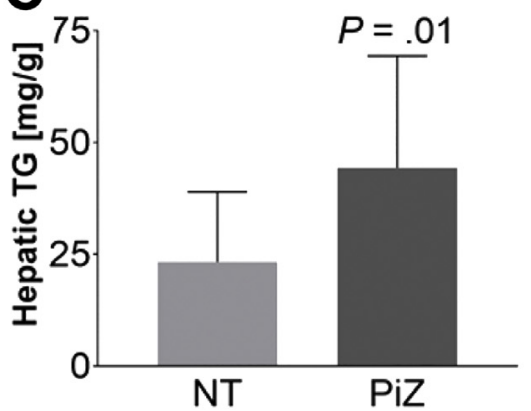

NT $P=.023$

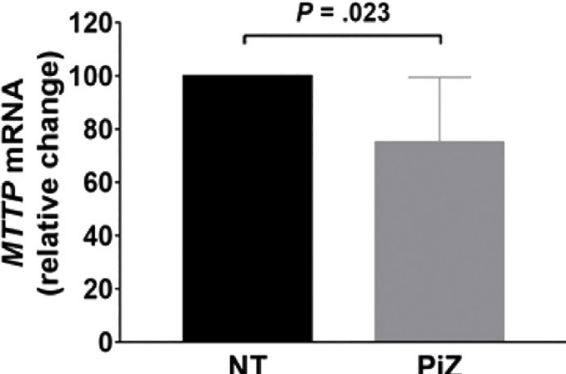

NT

PiZ

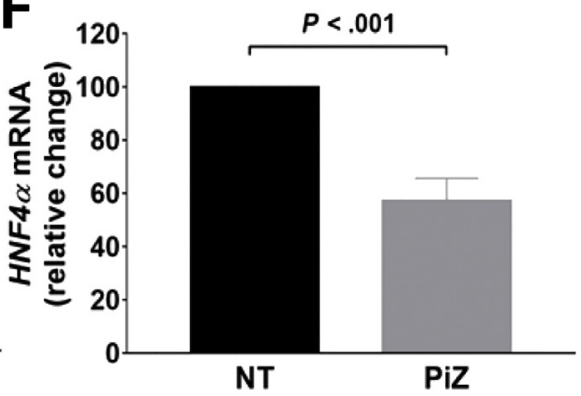

$\mathrm{PiZ}$
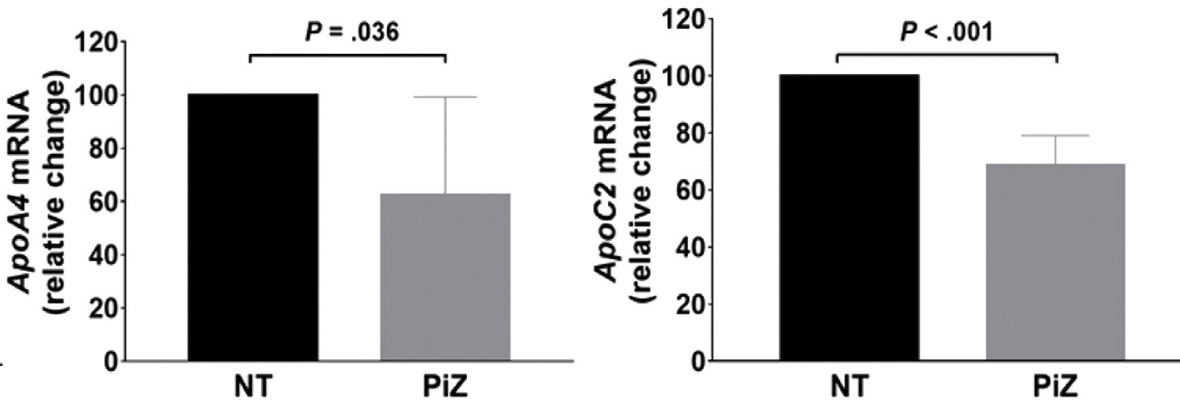

PiZ

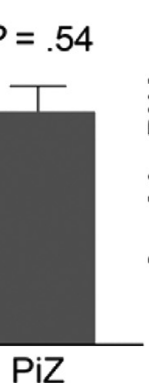

Figure 4. Extent of liver steatosis and expression of steatosis-related genes in untreated mice overexpressing the $A A T$ Pi $\mathrm{Z}$ variant (PiZ) and their non-transgenic littermates (NT). Fourteen transgenic, Pi`Z-overexpressing mice and 14 NT littermates, all 8-12 weeks of age, were histologically assessed for the extent of liver steatosis. $(A)$ Representative images of liver sections stained with H\&E (upper panel) or Oil Red O (ORO, lower panel). Scale bars $=20 \mu \mathrm{m}$. (B) Morphometric quantification of ORO staining. $(C)$ Hepatic triglyceride content was determined biochemically. Serum cholesterol levels $(E)$ and serum triglyceride levels $(D)$ in $\mathrm{Pi}^{\star} \mathrm{Z}$-overexpressing mice and their non-transgenic littermates. $(F)$ The relative expression of hepatic nuclear factor $4 \alpha$ (HNF4 $\alpha$ ), apolipoprotein A4 (ApoA4), microsomal triglyceride transfer protein (MTTP), and apolipoprotein C2 (ApoC2) were analyzed via reverse transcription polymerase chain reaction. $\mathrm{L} 7$ (mouse ribosomal protein) gene was used as a loading control. Mean expression in NT littermates was arbitrarily set as $100 \%$ and all other levels represent a ratio. chol., cholesterol; $\mathrm{PiZ}$, mice overexpressing the human AAT $\mathrm{Pi}^{\star} \mathrm{Z}$ variant; TG, triglycerides. 
particular, CAT values had a moderate correlation with age $(r=.42, P<.001)$ (Supplementary Figure $2 A)$ and the need for LTOT was more prevalent in older Pi*ZZ carriers (Supplementary Figure $2 B$ ). In contrast, although LSM $\geq 7.1 \mathrm{kPa}$ were less frequent in $\mathrm{Pi}^{*} \mathrm{ZZ}$ carriers $<50$ years of age, no relevant correlation between age and LSM was seen (Supplementary Figure $2 C$ and $D$ and Supplementary Table 6). A comparably weak relation with age distribution was noted both for APRI and HepaScore (Supplementary Figure $2 E-H$ and Supplementary Table 6).

To assess a potential association between lung disease and liver fibrosis, we compared CAT values with the 3 available non-invasive fibrosis scores. No significant correlation between CAT scores and LSM or APRI values were found (Figure $5 A$ and $C$, Supplementary Figure $3 A-D$ ). In contrast, HepaScore and CAT had a weak, but significant, correlation (Figure $5 E$, Supplementary Figure $3 E$ and $F$ ), which is likely due to the fact that HepaScore includes age as one parameter and because of that, older patients, who typically have a worse lung function, also harbor higher HepaScores.

As the presence of advanced $\mathrm{Pi}^{*} \mathrm{ZZ}$-related lung disease with altered right heart function may affect LSM values, we analyzed the results of non-invasive liver fibrosis assessments in individuals with and without need for LTOT. Both groups had similar LSM (Figure 5B). Moreover, the observed correlations among LSM, APRI, and HepaScore were similar in Pi*ZZ patients with and without LTOT (Supplementary Figure 4), thereby, clearly indicating that the presence of advanced lung disease did not compromise the usefulness of LSM. Similarly to LSM, neither APRI nor HepaScore values differed significantly in Pi*ZZ carriers with and without LTOT (Figure $5 D$ and $F$ ). Collectively, using 3 different non-invasive liver fibrosis tests, we demonstrated that Pi*ZZ carriers had no significant correlation between the burden of lung and liver disease.

Next, we studied the association between sex and liver-related parameters. Male Pi*ZZ carriers displayed higher sex-specific ALT, GGT, and bilirubin serum activities than female $\mathrm{Pi}^{*} \mathrm{ZZ}$ carriers $(86 \%$ vs $72 \%$ of ULN, $115 \%$ vs $81 \%$ of ULN, $59 \%$ vs $44 \%$ of ULN, respectively; all, $P<.05$ ) (Supplementary Table 7). Moreover, Pi*ZZ men had higher LSM, APRI, HepaScore, and CAP values compared to their counterparts $(7.8 \pm 7.0 \mathrm{kPa}$ vs $5.5 \pm$ $3.5 \mathrm{kPa}, 0.49 \pm 0.45$ units vs $0.30 \pm 0.18$ units, $0.54 \pm$ 0.32 units vs $0.30 \pm 0.27$ units, and $277 \pm 59 \mathrm{~dB} / \mathrm{m}$ vs $254 \pm 52 \mathrm{~dB} / \mathrm{m}$, respectively; all, $P<.001$ ) (Supplementary Table 7). Accordingly, Pi*ZZ men displayed a markedly higher risk for LSM $\geq 7.1 \mathrm{kPa}$ (unadjusted OR, 3.9; 95\% CI, 2.3-6.7) (Supplementary Table 8 and Supplementary Figure 5A), APRI $\geq 0.50$ units (unadjusted OR, 5.7; 95\% CI, 2.9-10.9) (Supplementary Table 9 and Supplementary Figure 6A), and HepaScore $\geq 0.48$ units (unadjusted OR, 4.1; 95\% CI, 2.3-7.4) (Supplementary Table 10 and Supplementary Figure $7 A$ ). Similarly, men had lower platelet counts and higher international normalized ratio values $(214.7 \pm 59.7 \mathrm{G} / \mathrm{L}$ vs
$253.1 \pm 69.7 \mathrm{G} / \mathrm{L}$, and $1.04 \pm 0.09$ vs $0.99 \pm 0.08$, respectively; both, $P<.001$ ) (Supplementary Table 7). All comparisons remained significant in a multivariable analysis (all, $P<.01$ ) (Supplementary Table 7 and not shown).

Together, male sex is associated with a more prominent liver disease, while age has no, or a less obvious, effect. Moreover, the extents of lung and liver disease did not correlate with each other.

\section{Additional Factors Associated With the Presence of Significant Liver Fibrosis}

Obese Pi*ZZ carriers, defined as body mass index $\geq 30$ $\mathrm{kg} / \mathrm{m}^{2}$, were more likely to display LSM $\geq 7.1 \mathrm{kPa}$ (Supplementary Table 8 and Supplementary Figure $5 B$ ), but neither to have APRI $\geq 0.50$ units (Supplementary Table 9 and Supplementary Figure $6 B$ ) nor HepaScore $\geq 0.48$ units (Supplementary Table 10 and Supplementary Figure $7 B$ ). AST and GGT serum levels were higher and platelet counts were lower in $\mathrm{Pi}^{*} \mathrm{ZZ}$ carriers with vs without $\mathrm{LSM} \geq 7.1 \mathrm{kPa}$ (Supplementary Table 8), APRI $\geq 0.50$ units vs $<0.50$ units (Supplementary Table 9), and HepaScore $\geq 0.48$ units vs $<0.48$ units (Supplementary Table 10). Elevated GGT (ie, above the sex-specific ULN) conferred a particularly strong risk to have $\mathrm{LSM} \geq 7.1 \mathrm{kPa}$ (unadjusted OR, 5.1; 95\% CI, 3.18.4) (Supplementary Table 8 and Supplementary Figure 5C) and APRI $\geq 0.50$ units (unadjusted OR, 5.1; 95\% CI, 3.0-8.7) (Supplementary Table 9 and Supplementary Figure 6C). Similarly, reduced platelets (ie, platelet count $<150 \mathrm{G} / \mathrm{L}$ ) markedly predisposed to $\mathrm{LSM} \geq 7.1 \mathrm{kPa}(\mathrm{OR}, 5.7 ; 95 \% \mathrm{CI}$, 2.7-11.8) (Supplementary Table 8 and Supplementary Figure $5 D$ ), APRI $\geq 0.50$ units (OR, 31.0; $95 \% \mathrm{CI}, 12.2-$ 79.0) (Supplementary Table 9 and Supplementary Figure $6 D$ ), and HepaScore $\geq 0.48$ units (OR, 5.2; 95\% CI, 1.8-15.3) (Supplementary Table 10 and Supplementary Figure $7 D$ ).

As detailed here, we demonstrated that demographic parameters as well as laboratory values help to estimate the individual risk for presence of significant liver fibrosis and may therefore be used in patient counseling. To facilitate that, we determined the frequency of elevated non-invasive liver fibrosis tests in clinically relevant $\mathrm{Pi}^{*} \mathrm{ZZ}$ subpopulations (Figure 6). These data revealed a low risk of significant liver fibrosis (ie, $L S M \geq 7.1 \mathrm{kPa}$, APRI $\geq 0.50$ units, and HepaScore $\geq 0.48$ units) in young women as well as women with normal serum GGT, AST, or ALT. Males younger than 50 years old constituted the male subpopulation with the lowest likelihood of increased liver fibrosis test values. Among serum parameters, elevated serum ALT, AST, or GGT was associated with higher occurrence of fibrosis test values suggestive of significant liver fibrosis. Pi*ZZ carriers with thrombocytopenia had, regardless of their sex, a high likelihood of significant liver fibrosis.

Collectively, our study uncovered that male sex; age older than 50 years; elevated ALT, AST, and GGT; and reduced platelet count associated with non-invasive liver fibrosis tests suggest the presence of significant fibrosis. 
Figure 5. Relationship between lung function and liver fibrosis in homozygous carriers of the AAT $\mathrm{Pi}^{\star} \mathrm{Z}$ variant $\left(\mathrm{Pi}^{\star} \mathrm{ZZ}\right)$. Four hundred and three $\mathrm{Pi}^{\star} \mathrm{ZZ}$ carriers were analyzed by 3 independent, noninvasive liver fibrosis tests (TE [FibroScan], APRI, and HepaScore), while the lung function was estimated via the need for LTOT, as well as CAT (a measure of lung function-related quality of life). Scatter plots display the values of non-invasive fibrosis scores in patients with different CAT values $(A, C$, and $E)$, as well as individuals with/without LTOT $(B, D$, and $F)$.
A
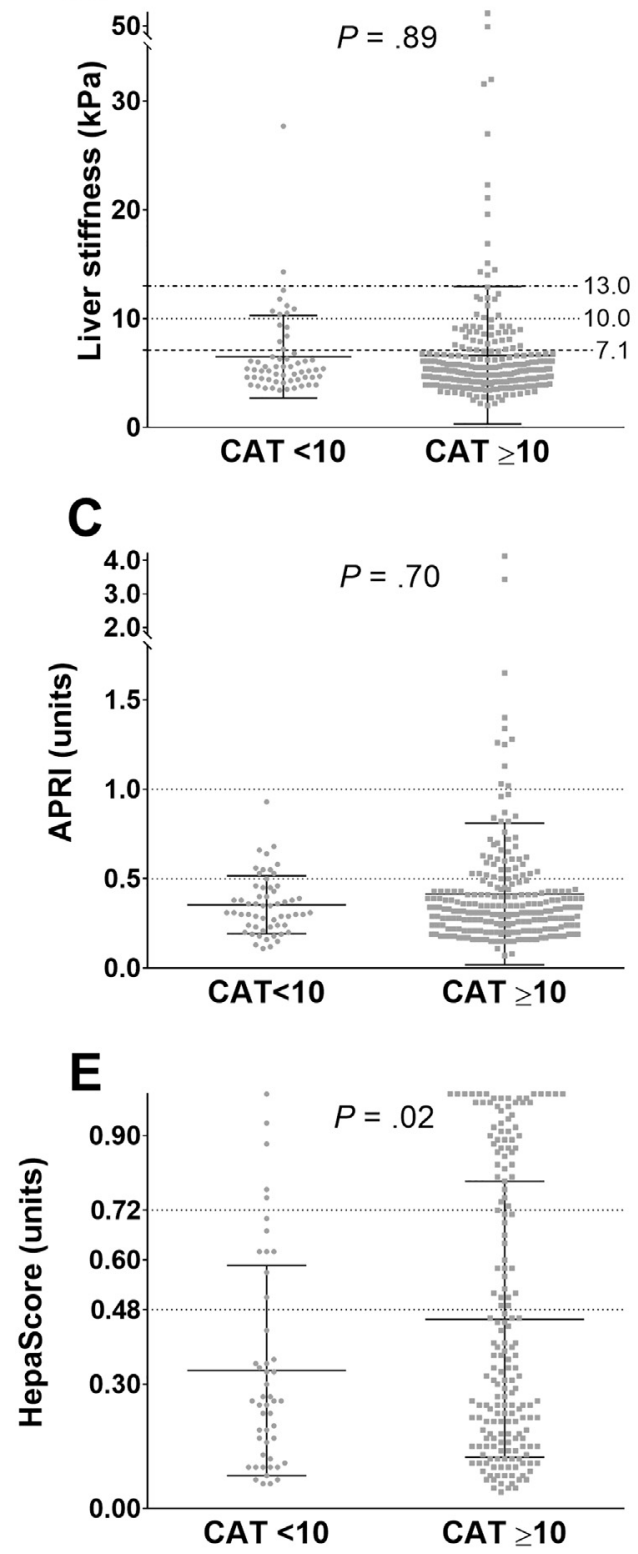

B
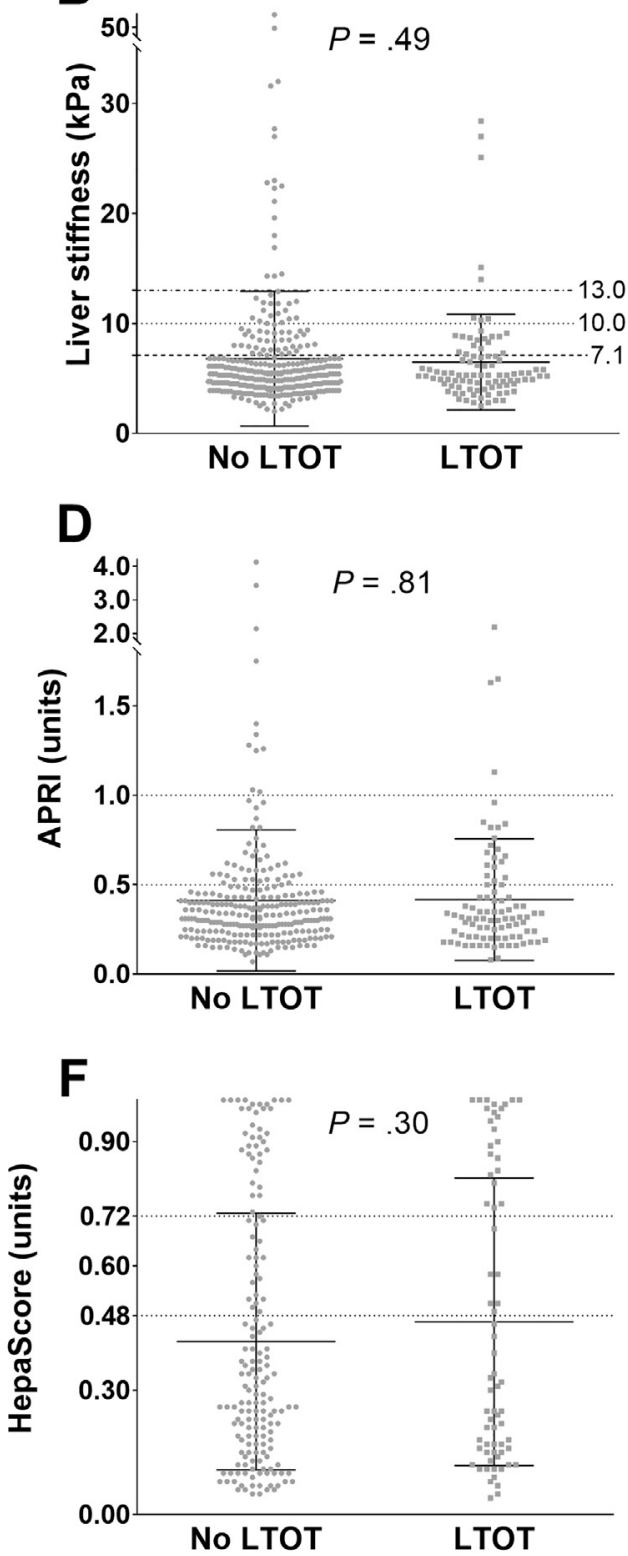

\section{Discussion}

Our study represents, to our knowledge, the largest systematic evaluation of the liver phenotype in $\mathrm{Pi}^{*} \mathrm{ZZ}$ carriers and the associated clinical contexts. We used 3 independent, non-invasive fibrosis tests that, compared to liver biopsy, are more suitable for long-term disease monitoring ${ }^{26,27}$ and for recruitment of a real-life population, including individuals without clinically relevant liver involvement.

Using these tests, the presence of significant liver fibrosis was suggested in $20 \%-36 \%$ and the presence of advanced liver fibrosis in $5 \%-26 \%$ of Pi*ZZ study participants. Moreover, $\mathrm{Pi}$ ZZ carriers had 9-20 times higher odds than non-carriers to have liver fibrosis test results that indicate advanced liver fibrosis. While these numbers clearly document the vulnerability of $\mathrm{Pi}^{*} \mathrm{ZZ}$ carriers for liver disease, the frequencies are lower than former reports suggesting that up to $43 \%$ of $\mathrm{Pi}^{*} \mathrm{ZZ}$ individuals may develop cirrhosis, ${ }^{22,38}$ or a recent TE-biopsy study suggesting that $35 \%$ have significant fibrosis. Additionally, the observed risk for presence of advanced liver fibrosis appears lower than the reported odds of $\mathrm{Pi}^{*} \mathrm{ZZ}$ subjects to receive liver transplantation. ${ }^{39}$

These differences are not surprising, given the limited size of the previously analyzed cohorts and potential bias that may arise due to the recruitment via liver biopsy. Additionally, given that the occurrence of $\mathrm{Pi}^{*} \mathrm{Z}$ variant seems to promote the decompensation of end-stage liver disease, ${ }^{40}$ the odds requiring a liver transplantation may surpass the risk to form advanced liver fibrosis. On the other hand, the careful screening for hepatic comorbidities and exclusion of individuals with previously existing liver disease and those 

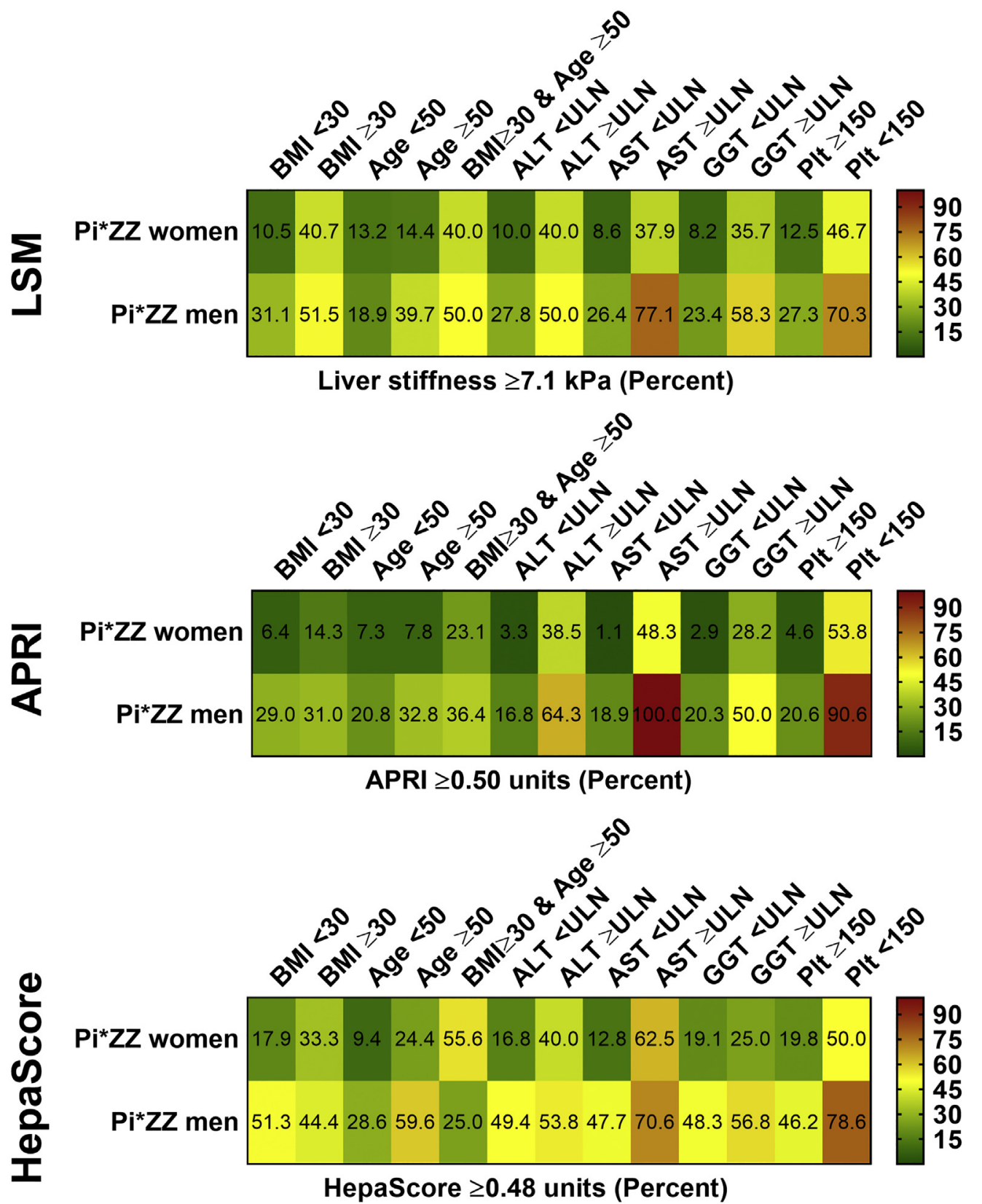

Figure 6. Rate of $\mathrm{Pi}^{\star} \mathrm{ZZ}$ carriers with significant liver fibrosis according to LSM, APRI, and HepaScore among specific subpopulations. Relative frequencies (\%) are shown and visualized by a color coding (right panel). The cutoffs for liver stiffness (7.1 kPa), APRI (0.50 units), and HepaScore $(0.48$ units) represent established cutoffs suggesting the presence of significant fibrosis (F2). Plt, platelets.

with potentially harmful alcohol consumption may have led to underestimation of the liver disease burden occurring in the general population. While the current article describes the baseline evaluation of the study participants, a longitudinal follow-up is being carried out to determine the rate of liver fibrosis development, hepatic decompensation, or hepatocellular carcinoma formation.

A limitation of our study is that the extent of liver fibrosis was evaluated by non-invasive methods only. To minimize the associated risks, we used 3 independent tests and carried out an extensive cross-validation that helped us to address potential caveats. For example, TE values tended to be higher in obese individuals, whereas the other fibrosis tests did not reveal an obvious relationship between both parameters. This is in line with the observation that TE may overestimate the extent of liver fibrosis in overweight individuals. ${ }^{41}$ On the other hand, previous studies support the TE findings in that they suggested a promoting effect of obesity on liver disease development in Pi*ZZ carriers. ${ }^{42,43}$

Another limitation of LSM, which has been carefully addressed in our study, is its potential affection by hepatic congestion (eg, caused by right-sided heart failure). ${ }^{44}$ Although we cannot fully exclude this possibility in selected patients, several analytical outcomes argue against this as a major confounding factor. In particular, we could not detect any differences in LSM between Pi*ZZ carriers with and without LTOT (Figure 5), despite the fact that the need for LTOT is commonly associated with right-sided heart failure. Moreover, the correlation between the applied fibrosis tests was similarly robust in patients with 
and without need for LTOT (Supplementary Figure 4). Finally, there was no obvious relationship between LSM and CAT values (Figure 5 and Supplementary Figure 3), even though CAT constitutes a well-validated measure of lung function-related quality of life. The parallel assessment of TE, APRI, and HepaScore values revealed a moderate correlation between the former 2, whereas the correlations with the HepaScore were less robust. Accordingly, previous studies demonstrated that $\alpha 2$-macroglobulin, a component of the HepaScore, is elevated in Pi*ZZ carriers. ${ }^{45}$ This finding, together with the fact that the HepaScore had a weaker association with serum liver enzymes than the other scores, suggests that it might be a suboptimal measure of liver fibrosis in this population.

CAP indicated that $\mathrm{Pi}^{*} \mathrm{ZZ}$ carriers have more pronounced liver steatosis, which is in line with a published liver biopsy series of $\mathrm{Pi}^{*} \mathrm{ZZ}$ adults. ${ }^{46}$ The simultaneous presence of decreased triglyceride and very-low-density lipoprotein cholesterol levels suggest that $\mathrm{Pi}^{*} \mathrm{Z}$ accumulation in the endoplasmic reticulum affects lipid metabolism of this subcompartment. This hypothesis was in part strengthened by our murine experiments suggesting that intrahepatocytic $\mathrm{Pi}^{*} \mathrm{Z}$ accumulation might result in increased hepatic triglyceride content. In particular, an impaired hepatocyte nuclear factor $4 \alpha$ signaling observed in $\mathrm{Pi}^{*} \mathrm{Z}$-overexpressing mice may result in impaired lipoprotein assembly and lipid secretion. ${ }^{36,37}$ An alternative explanation is that hepatic steatosis arises from proteotoxic stress, a known result of intrahepatocytic $\mathrm{Pi}^{*} \mathrm{Z}$ accumulation. ${ }^{47-49}$ In this respect, an overwhelmed lipid autophagy due to the need to degrade excess $\mathrm{Pi}^{*} \mathrm{Z}$, as well as the reported interaction of $\mathrm{Pi}^{*} \mathrm{Z}$ with lipoprotein particles might be of relevance. ${ }^{50,51}$ Therefore, further studies are needed to delineate the precise factors contributing to hepatic steatosis in both Pi*Zoverexpressing mice and $\mathrm{Pi}^{*} \mathrm{ZZ}$ humans.

With regard to monitoring individual $\mathrm{Pi}^{*} \mathrm{ZZ}$ carriers, our data corroborate and extend the following observations: irrespective of a Pi*ZZ carrier's age, liver enzymes are elevated in only a minority of them ${ }^{24,25}$; and males are at a higher risk for an advanced liver disease developing than females (Figure $3 A$ ). ${ }^{22,52}$ With regard to the latter, murine data demonstrate that males synthesize more AAT and that testosterone treatment of female $\mathrm{Pi}^{*} \mathrm{Z}$-overexpressing mice increases AAT expression. ${ }^{53}$ Hence, in case of $\mathrm{Pi}^{*} \mathrm{Z}$ mutation, increased $\mathrm{Pi}^{*} \mathrm{Z}$ synthesis might result in progressive fibrogenesis.

Our evaluation of $\mathrm{Pi}^{*} \mathrm{ZZ}$ carriers also yielded the following novel and less expected findings:

1. Unlike what has been reported previously, ${ }^{54,55}$ we did not see a relevant correlation between the severity of lung and liver involvement (Figure 5). While APRI and TE did not correlate with lung function significantly, a weak correlation with HepaScore might be due to age, a component of the HepaScore, as a confounding factor.

2. Age younger than 50 years was associated with lower rates of significant liver fibrosis in males but, other than what has been reported previously, ${ }^{5,23}$ age younger than 50 years had no major impact in females. This finding most likely explains why there is no obvious linear correlation between liver fibrosis and age (Supplementary Figure $2 C-F$ ).

3. Among routinely assessed laboratory parameters, elevated GGT levels were particularly predictive for the presence of liver fibrosis (Supplementary Figures 5-7C). This is not surprising because GGT causally associates with obesity and plays an important role in defense against oxidative stress and is therefore an established marker of metabolic liver disease. $^{56,57}$ Similarly, the observed usefulness of platelet count (Supplementary Figures 5-7D) is well in line with its known association with extent of portal hypertension. ${ }^{58}$

4. In contrast to a previous report, ALT, AST, and GGT reasonably well discriminated between patients with and without significant liver fibrosis, and when within normal range, might be particularly useful to identify females at low risk of fibrosis. On the other hand, they are not sufficient to rule out significant fibrosis in males (Figure 6).

In summary, our large, international cohort defined the liver phenotype of $\mathrm{Pi}^{*} \mathrm{ZZ}$ adults and uncovered associated metabolic alterations. The high odds of $\mathrm{Pi}^{*} \mathrm{ZZ}$ carriers to develop a clinically relevant liver affection warrant a regular assessment of liver enzymes and of liver fibrosis. As liver enzymes were regularly determined in only $45 \%$ of our Pi*ZZ study participants, and as liver fibrosis was assessed in a negligible minority only, the presented data should raise awareness for this neglected condition. Simultaneous use of 3 non-invasive fibrosis tests clearly delineated the usefulness of commonly available laboratory values, including AST, GGT, and platelets, in the initial assessment of the Pi*ZZ liver phenotype. In addition, a calculation of APRI provides another widely available tool in the assessment of these individuals. The ongoing, longitudinal evaluation of this largest prospective $\mathrm{Pi}^{*} \mathrm{ZZ}$ cohort will be useful to gain further insights into the process of disease development.

\section{Supplementary Material}

Note: To access the supplementary material accompanying this article, visit the online version of Gastroenterology at www.gastrojournal.org, and at https://doi.org/10.1053/ j.gastro.2019.05.013.

\section{References}

1. Carrell RW, Lomas DA. Alpha1-antitrypsin deficiency-a model for conformational diseases. N Engl J Med 2002; 346:45-53.

2. Dickens JA, Lomas DA. Why has it been so difficult to prove the efficacy of alpha-1-antitrypsin replacement therapy? Insights from the study of disease pathogenesis. Drug Des Devel Ther 2011;5:391-405. 
3. Strnad P, Nuraldeen R, Guldiken N, et al. Broad spectrum of hepatocyte inclusions in humans, animals, and experimental models. Compr Physiol 2013;3:1393-1436.

4. Silverman EK, Sandhaus RA. Alpha1-antitrypsin deficiency. N Engl J Med 2009;360:2749-2757.

5. Greene CM, Marciniak SJ, Teckman J, et al. Alpha1antitrypsin deficiency. Nat Rev Dis Primers 2016; 2:16051.

6. Sifers RN. Intracellular processing of alpha1-antitrypsin. Proc Am Thorac Soc 2010;7:376-380.

7. Perlmutter DH. Autophagic disposal of the aggregationprone protein that causes liver inflammation and carcinogenesis in alpha-1-antitrypsin deficiency. Cell Death Differ 2009;16:39-45.

8. Lomas DA, Evans D, Finch JT, et al. The mechanism of $Z$ alpha1-antitrypsin accumulation in the liver. Nature 1992; 357:605-607.

9. Lomas DA, Hurst JR, Gooptu B. Update on alpha-1 antitrypsin deficiency: new therapies. J Hepatol 2016; 65:413-424.

10. Chapman KR, Burdon JG, Piitulainen E, et al. Intravenous augmentation treatment and lung density in severe alpha1 antitrypsin deficiency (RAPID): a randomised, double-blind, placebo-controlled trial. Lancet 2015; 386:360-368.

11. Crystal RG. Compelling evidence for the efficacy of alpha1-antitrypsin augmentation treatment for alpha1antitrypsin deficiency. Lancet Respir Med 2017;5:7-8.

12. Teckman JH, Blomenkamp KS. Pathophysiology of alpha-1 antitrypsin deficiency liver disease. Methods Mol Biol 2017;1639:1-8.

13. Perlmutter DH. Alpha-1-antitrypsin deficiency: importance of proteasomal and autophagic degradative pathways in disposal of liver disease-associated protein aggregates. Annu Rev Med 2011;62:333-345.

14. Sveger T. Liver disease in alpha1-antitrypsin deficiency detected by screening of 200,000 infants. N Engl J Med 1976;294:1316-1321.

15. Wall M, Moe E, Eisenberg J, et al. Long-term follow-up of a cohort of children with alpha-1-antitrypsin deficiency. J Pediatr 1990;116:248-251.

16. Teckman JH. Alpha1-antitrypsin deficiency in childhood. Semin Liver Dis 2007;27:274-281.

17. Townsend SA, Edgar RG, Ellis PR, et al. Systematic review: the natural history of alpha-1 antitrypsin deficiency, and associated liver disease. Aliment Pharmacol Ther 2018;47:877-885.

18. Wanner A, Groft SC, Teagarden JR, et al. Clinical trial design for alpha-1 antitrypsin deficiency: a model for rare diseases. Chronic Obstr Pulm Dis 2015;2:177-190.

19. Heneghan MA, Yeoman AD, Verma S, et al. Autoimmune hepatitis. Lancet 2013;382:1433-1444.

20. Hirschfield GM, Karlsen TH, Lindor KD, et al. Primary sclerosing cholangitis. Lancet 2013;382:1587-1599.

21. Ala A, Walker AP, Ashkan K, et al. Wilson's disease. Lancet 2007;369:397-408.

22. Eriksson S, Carlson J, Velez R. Risk of cirrhosis and primary liver cancer in alpha 1-antitrypsin deficiency. N Engl J Med 1986;314:736-739.
23. Mostafavi B, Diaz S, Tanash HA, et al. Liver function in alpha-1-antitrypsin deficient individuals at 37 to 40 years of age. Medicine (Baltimore) 2017;96:e6180.

24. Clark VC, Marek G, Liu C, et al. Clinical and histologic features of adults with alpha- 1 antitrypsin deficiency in a non-cirrhotic cohort. J Hepatol 2018;69: 1357-1364.

25. Clark VC, Dhanasekaran R, Brantly M, et al. Liver test results do not identify liver disease in adults with alpha(1)-antitrypsin deficiency. Clin Gastroenterol Hepatol 2012;10:1278-1283.

26. Friedrich-Rust M, Poynard T, Castera L. Critical comparison of elastography methods to assess chronic liver disease. Nat Rev Gastroenterol Hepatol 2016;13: 402-411.

27. Tapper EB, Lok AS. Use of liver imaging and biopsy in clinical practice. N Engl J Med 2017;377:756-768.

28. Karlas T, Petroff D, Sasso M, et al. Individual patient data meta-analysis of controlled attenuation parameter (CAP) technology for assessing steatosis. J Hepatol 2017;66:1022-1030.

29. Wai CT, Greenson JK, Fontana RJ, et al. A simple noninvasive index can predict both significant fibrosis and cirrhosis in patients with chronic hepatitis C. Hepatology 2003;38:518-526.

30. Adams LA, Bulsara M, Rossi E, et al. Hepascore: an accurate validated predictor of liver fibrosis in chronic hepatitis C infection. Clin Chem 2005;51:1867-1873.

31. Xiao G, Yang J, Yan L. Comparison of diagnostic accuracy of aspartate aminotransferase to platelet ratio index and fibrosis-4 index for detecting liver fibrosis in adult patients with chronic hepatitis B virus infection: a systemic review and meta-analysis. Hepatology 2015; 61:292-302.

32. Chou R, Wasson N. Blood tests to diagnose fibrosis or cirrhosis in patients with chronic hepatitis $C$ virus infection: a systematic review. Ann Intern Med 2013;158: 807-820.

33. Huang $Y$, Adams LA, Joseph J, et al. The ability of Hepascore to predict liver fibrosis in chronic liver disease: a meta-analysis. Liver Int 2017;37:121-131.

34. Vilar-Gomez E, Chalasani N. Non-invasive assessment of non-alcoholic fatty liver disease: clinical prediction rules and blood-based biomarkers. J Hepatol 2018; 68:305-315.

35. Carlson JA, Rogers BB, Sifers RN, et al. Accumulation of $\mathrm{PiZ}$ alpha 1-antitrypsin causes liver damage in transgenic mice. J Clin Invest 1989;83:1183-1190.

36. Hayhurst GP, Lee YH, Lambert G, et al. Hepatocyte nuclear factor 4 alpha (nuclear receptor 2A1) is essential for maintenance of hepatic gene expression and lipid homeostasis. Mol Cell Biol 2001;21:1393-1403.

37. Piccolo P, Annunziata P, Soria LR, et al. Down-regulation of hepatocyte nuclear factor-4alpha and defective zonation in livers expressing mutant $Z$ alpha1-antitrypsin. Hepatology 2017;66:124-135.

38. Elzouki AN, Eriksson S. Risk of hepatobiliary disease in adults with severe alpha 1-antitrypsin deficiency (PiZZ): is chronic viral hepatitis B or C an additional risk factor 
for cirrhosis and hepatocellular carcinoma? Eur J Gastroenterol Hepatol 1996;8:989-994.

39. Adam R, Karam V, Delvart V, et al. Evolution of indications and results of liver transplantation in Europe. A report from the European Liver Transplant Registry (ELTR). J Hepatol 2012;57:675-688.

40. Schaefer B, Mandorfer M, Viveiros A, et al. Heterozygosity for the alpha-1-antitrypsin Z-allele in cirrhosis is associated with more advanced disease. Liver Transpl 2018;24:744-751.

41. Bazerbachi F, Haffar S, Wang Z, et al. Range of normal liver stiffness and factors associated with increased stiffness measurements in apparently healthy individuals. Clin Gastroenterol Hepatol 2019;17:54-64.e1.

42. Williams R, Aspinall R, Bellis M, et al. Addressing liver disease in the UK: a blueprint for attaining excellence in health care and reducing premature mortality from lifestyle issues of excess consumption of alcohol, obesity, and viral hepatitis. Lancet 2014;384: 1953-1997.

43. Stender S, Kozlitina J, Nordestgaard BG, et al. Adiposity amplifies the genetic risk of fatty liver disease conferred by multiple loci. Nat Genet 2017;49:842-847.

44. Lemmer A, VanWagner LB, Ganger D. Assessment of advanced liver fibrosis and the risk for hepatic decompensation in patients with congestive hepatopathy. Hepatology 2018;68:1633-1641.

45. Kotke V, Wiedmann S, Nell C, et al. [Alpha-2 macroglobulin serum level in patients with alpha-1 antitrypsin deficiency]. Pneumologie 2017;71:580-586.

46. Dawwas MF, Davies SE, Griffiths WJ, et al. Prevalence and risk factors for liver involvement in individuals with PiZZ-related lung disease. Am J Respir Crit Care Med 2013;187:502-508.

47. Kuscuoglu D, Janciauskiene S, Hamesch K, et al. Liver-master and servant of serum proteome. J Hepatol 2018;69:512-524.

48. Malhi H, Kaufman RJ. Endoplasmic reticulum stress in liver disease. J Hepatol 2011;54:795-809.

49. Baiceanu A, Mesdom P, Lagouge M, et al. Endoplasmic reticulum proteostasis in hepatic steatosis. Nat Rev Endocrinol 2016;12:710-722.

50. Diffenderfer MR, Schaefer EJ. The composition and metabolism of large and small LDL. Curr Opin Lipidol 2014;25:221-226.

51. Madrigal-Matute J, Cuervo AM. Regulation of liver metabolism by autophagy. Gastroenterology 2016; 150:328-339.

52. Teckman JH, Qu D, Perlmutter DH. Molecular pathogenesis of liver disease in alpha1-antitrypsin deficiency. Hepatology 1996;24:1504-1516.

53. Rudnick DA, Liao Y, An JK, et al. Analyses of hepatocellular proliferation in a mouse model of alpha-1-antitrypsin deficiency. Hepatology 2004;39: 1048-1055.
54. Crystal RG. Alpha 1-antitrypsin deficiency, emphysema, and liver disease. Genetic basis and strategies for therapy. J Clin Invest 1990;85:1343-1352.

55. Stolk J, Seersholm N, Kalsheker N. Alpha1-antitrypsin deficiency: current perspective on research, diagnosis, and management. Int $\mathrm{J}$ Chron Obstruct Pulmon Dis 2006;1:151-160.

56. Kunutsor SK. Gamma-glutamyltransferase-friend or foe within? Liver Int 2016;36:1723-1734.

57. Fall T, Hagg S, Magi R, et al. The role of adiposity in cardiometabolic traits: a Mendelian randomization analysis. PLoS Med 2013;10:e1001474.

58. Berzigotti A, Seijo S, Arena U, et al. Elastography, spleen size, and platelet count identify portal hypertension in patients with compensated cirrhosis. Gastroenterology 2013;144:102111.e1.

Author names in bold designate shared co-first authorship.

Received May 19, 2018. Accepted May 9, 2019.

Reprint requests

Address requests for reprints to: Pavel Strnad, MD, Coordinating Center for Alpha1-Antitrypsin Deficiency-Related Liver Disease of the European Reference Network "Rare Liver" and the European Association for the Study of the Liver Registry Group "Alpha1-Liver", University Hospital Aachen, Pauwelsstrasse 30, 52074 Aachen, Germany. e-mail: pstrnad@ukaachen.de.

\section{Acknowledgments}

The authors thank the national patient organizations (ie, Alpha1 Deutschland: Marion Wilkens and Gabi Niethammer, Alpha1 Oesterreich: Ella Geiblinger and Christa Berger, Alpha1 Plus Belgium: Frank Willersinn, Alpha1 Nederland: Heleen Groen, Alfa-1 Denmark: Gunhil Norhave, as well as Alfa1 Spain and Alfa1 Portugal) for their help with the execution of our study. Moreover, we thank Flavio Pereira and Mariana Cardoso for their help with recruiting patients. We also thank all patients for their participation in our study. Author contributions: Study concept and design: K.H., P.S. Acquisition of data: K.H., M.M., V.M.P., L.S.M., M.P., G.E.D., M.C.R., C.V.H., V.W., J.V., C.L., M.F., I.S., N.G., B.Z., A.A., B. Schaefer, H.Z., E.A., T.R., M.W., B. Siegmund, C.S., R.G., L.M., D.C., M.B.-M., J.v.H., E.Y., J.S., W.G., V.K., W.W., R.M., R.B., R.K., M.B., M.M., S.J., F.S., F.L., R.L., J.G., W.J.G., M.T., A.K., C.T., P.S. Analysis and interpretation of data: K.H., C.V.H., V.W., J.V., E.Y., D.B., S.J., P.S. Drafting of the manuscript: K.H., P.S. Critical revision of the manuscript for important intellectual content: all authors. Figures and Tables: K.H., C.V.H., P.S. Statistical analysis: K.H., D.B. Obtained funding: K.H., M.M., J.G., F.L., M.T., A.K., C.T., P.S. Study supervision: K.H., P.S. All authors had full access to all of the data and approved the final version of this manuscript. All authors can take responsibility for the integrity of the data and the accuracy of the data analysis.

\section{Conflicts of interest}

The authors disclose no conflicts.

\section{Funding}

This work was supported by the European Association for the Study of the Liver registry grant on $\alpha 1$-antitrypsin-related liver disease, the Deutsche Forschungsgemeinschaft consortium SFB/TRR57 "Liver Fibrosis" (both to P.S. and C.T.), the Interdisciplinary Center for Clinical Research within the faculty of Medicine at the Rheinisch-Westfälische Technische Hochschule Aachen University (to P.S.), the Else Kroener Excellence Fellowship (to P.S.), an unrestricted research grant from Grifols (to P.S.), the German Liver Foundation (to K.H.), the German Gastroenterological Association (to K.H.), the START program within the medical faculty at RWTH Aachen University (to K.H.), the Liver Systems Medicine (BMBF LiSyM 031L0051; to F.L. and C.T.), the Joseph-Skoda Award of the Austrian Society of Internal Medicine (to M.M.), the European Union 7th framework program (/FP7/2007-2013, project "FLIP"; to M.T.), the Instituto de Salud Carlos III, Spain co-funded by European Union (European Regional Development Fund/European Socia Fund, "Investing in your future"; grants PI15/00066 and PI18/00947 to J.G.), and the Swiss National Funds (SNF no. 310030_169196; to F.S.). 


\section{Supplementary Methods}

\section{Ethical Statement}

Ethical approval was provided by the Institutional Review Board of Aachen University (EK 173/15), as well as by the Institutional Ethics Committees of the participating centers (ie, Homburg and Berlin from Germany; Vienna, Innsbruck, and Salzburg from Austria; Odense from Denmark; Madeira, Lisbon, and Porto from Portugal; Barcelona from Spain; Cambridge from UK; and Bern from Switzerland). The study was conducted according to the Declaration of Helsinki (Hong Kong Amendment), as well as Good Clinical Practice (European guidelines), and was registered with ClinicalTrials.gov (NCT02929940).

\section{Recruitment of Study Population}

Pi*ZZ carriers have been recruited during an awareness campaign (for details see above) that was supported via an AATD liver-related website (www.alpha1-liver.eu), a telephone hotline, a presence on social media, talks at patient meetings, as well as contributions to patient-centered periodicals in various countries. Additional carriers were attracted by the University Hospital Aachen as the coordinating center for AATD-related liver disease of 2 European initiatives (European Association for the Study of the Liver Registry Group "Alpha1-Liver" and European Reference Network "Rare-Liver"). For the exploratory cohort, both carriers and non-carriers had been recruited in the period from April 1, 2015 through September 30, 2017 in 7 different countries (Supplementary Table 1). For the confirmatory cohort, $\mathrm{Pi}^{*} \mathrm{ZZ}$ carriers had been recruited from October 1, 2017 through April 30, 2018 in four different countries (Supplementary Table 2).

Every participant filled out standardized questionnaires including information on demographic parameters, relevant comorbidities, and lung-related (eg, CAT, need for LTOT) and liver-related health (eg, surveillance and alcohol consumption).

\section{Assessment of Liver Disease}

In all participants, the presence of a previously diagnosed liver disease has been excluded by personal interview (ie, no established diagnosis of chronic liver disease and no history of liver resection or liver transplantation) and laboratory workup. Chronic viral hepatitis B and C were excluded serologically. Serum levels of ferritin and transferrin saturation were determined to evaluate the presence of hereditary hemochromatosis. For every individual patient, drinking habits were assessed in a face-to-face interview evaluating the long-term drinking habits and thereby the average weekly number of alcoholic drinks was determined. Consequently, the mean amount of alcohol consumed per week was calculated and used for further analysis. Patients with self-reported pathologic alcohol consumption ( $>40 \mathrm{~g} / \mathrm{d}$ for women and $>60 \mathrm{~g} / \mathrm{d}$ for men) have been excluded ( $5 \mathrm{Pi}^{*} \mathrm{ZZ}$ carriers and 1 non-carrier).
Finally, patients with elevated ALT or AST $>5 \times$ the sexspecific ULN or alkaline phosphatase $>2 \times$ the sex-specific ULN on the day of recruitment have been excluded ( 2 $\mathrm{Pi}^{*} \mathrm{ZZ}$ carriers and 0 non-carrier) because marked elevation of these parameters precludes a reliable assessment by TE. Isoelectric focusing and genotyping were carried out by the corresponding national AAT reference laboratories, as described. ${ }^{1}$

On the day of inclusion, venous blood was obtained, centrifuged, aliquoted, and stored at $-80^{\circ} \mathrm{C}$. Presence of hepatitis B surface antigen, anti-hepatitis $\mathrm{C}$ virus antibodies (Cobas e 601; Roche Diagnostics, Mannheim, Germany), and serum very-low-density lipoprotein cholesterol (Hydrasys, Sebia, Fulda, Germany) were determined by appropriate assays. $\alpha 2$-Macroglobulin (BN ProSpec; Siemens, Marburg, Germany) and hyaluronic acid (COBAS c501; Wako Chemicals, Neuss, Germany) were measured in the serum to calculate the HepaScore. All tests have been approved for use in clinical routine.

\section{Pi*Z-Overexpressing Mice and Quantification of Hepatic Steatosis}

All mice were kept on standard diet in the animal facility of Rheinisch-Westfälische Technische Hochschule Aachen University (Aachen, Germany). Liver tissues were dissected as described previously. ${ }^{2}$ The extent of lipid accumulation was evaluated via Oil Red 0 staining. Briefly, liver specimens were embedded in Tissue-Tek Compound (Sakura, Torrance, CA), snap-frozen, and cut into $5-\mu \mathrm{m}$ thin sections. The sections were fixed in $10 \%$ formalin for 5 minutes at room temperature and rinsed first with tap water, then with $60 \%$ isopropanol (Sigma-Aldrich, St Louis, MO). Samples were incubated in fresh Oil Red 0 working solution for 15 minutes at room temperature and the unbound dye was removed with $60 \%$ isopropanol. The counterstaining was carried out with hematoxylin. The pictures were taken with the Zeiss light microscope ImagerA2 (Zeiss, Göttingen, Germany) and the amount of lipids was quantified with Image J software (National Institutes of Health, Bethesda, MD). To determine the hepatic triglyceride content, liver tissues were incubated in ethanolic $\mathrm{KOH}$ overnight at $55^{\circ} \mathrm{C}$. The resulting liver homogenates were mixed with a 1:1 EtOH/ $\mathrm{H}_{2} \mathrm{O}$ solution and centrifuged at $14,000 \mathrm{rpm}$ for 5 minutes to remove the debris. Then $1 \mathrm{M} \mathrm{MgCl}_{2}$ was added and the mixture was incubated on ice for 10 minutes. After additional centrifugation, the supernatants were collected and triglyceride amount was analyzed at the Department of Clinical Chemistry of Aachen University Hospital. The concentration was normalized to wet liver weight. Serum triglycerides and cholesterol levels were also analyzed at the Department of Clinical Chemistry of Aachen University Hospital. Animal handling was carried out in accordance with the German law for welfare of laboratory animals.

RNA was isolated from 2-month-old mouse livers via RNeasy mini isolation kit (Qiagen, Hilden, Germany). The RNA samples were translated to complementary DNA with M-MLV reverse transcriptase kit (Promega, Madison, WI). 
The relative expression of genes of interest was determined using specific primers for $H N F 4 \alpha$ (hepatocyte nuclear factor 4 $\alpha$, F: GGC CAA GAT TGA CAA CCT GC, R: TGA GAG GGC ATC GTG TTA GC), ApoA4 (apolipoprotein A-IV, F: ATG CCA AGG AGG CTG TAG AAC, R: AAA GGG CAC CAG CTT GTT GT), ApoC2 (apolipoprotein C-II, F: AAC CAG GAA GAT GAC TCG GG, R: AAA TGC CTG CGT AAG TGC TC), and Mttp (microsomal triglyceride transfer protein, F: AGA TGG ACG CCA GCT TTT GTT, R: TCC TTT GCC CCC ATC AAG AA). The messenger RNA expression was normalized to the levels of the housekeeping ribosomal gene $L 7$ (ribosomal protein, F:
GAA AGG CAA GGA GGA AGC TCA TCT, R: AAT CTC AGT GCG GTA CAT CTG CCT).

\section{Supplementary References}

1. Greulich T, Ottaviani S, Bals R, et al. Alpha1-antitrypsin deficiency-diagnostic testing and disease awareness in Germany and Italy. Respir Med 2013;107:1400-1408.

2. Guldiken N, Kobazi Ensari G, Lahiri P, et al. Keratin 23 is a stress-inducible marker of mouse and human ductular reaction in liver disease. J Hepatol 2016;65:552-559.
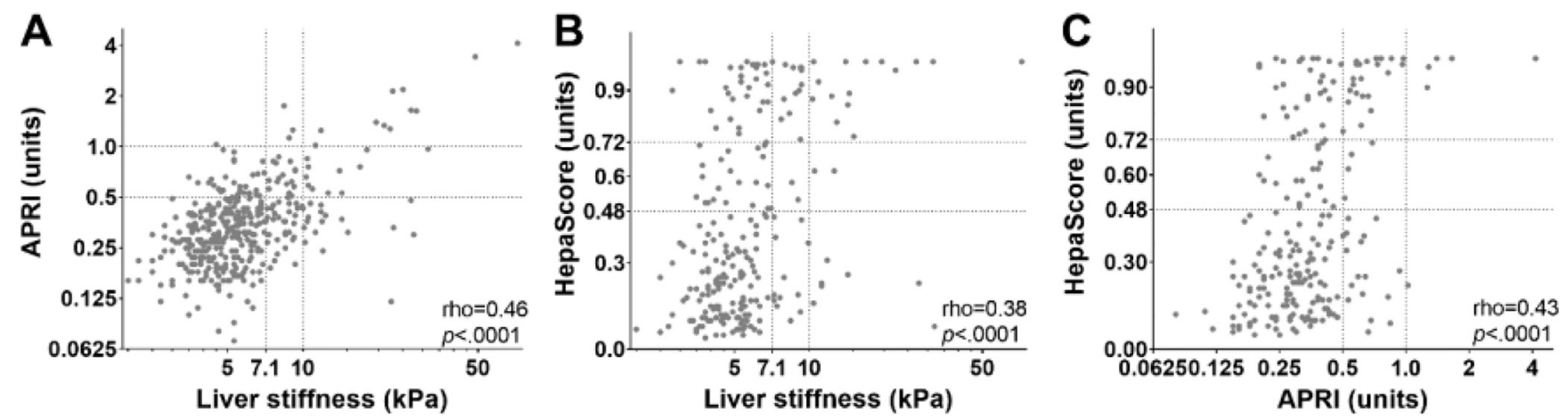

Supplementary Figure 1. Correlation of non-invasive liver fibrosis parameters in homozygous carriers of the $A A T P{ }^{\star} Z \mathrm{Z}$ variant $\left(\mathrm{Pi}^{\star} \mathrm{ZZ}\right)$. The dotted lines represent the proposed cutoffs for significant fibrosis (liver stiffness: $7.1 \mathrm{kPa}, \mathrm{APRl}: 0.50 \mathrm{units}$, HepaScore: 0.48 units) and advanced fibrosis (liver stiffness: $10 \mathrm{kPa}$, APRI 1.00, HepaScore: 0.72). (A) Correlation of liver stiffness $\left(\log _{10}\right)$ and APRI $\left(\log _{2}\right)$. (B) Correlation of liver stiffness $\left(\log _{10}\right)$ and HepaScore. $(C)$ Correlation of APRI (log $)$ and HepaScore. 
A

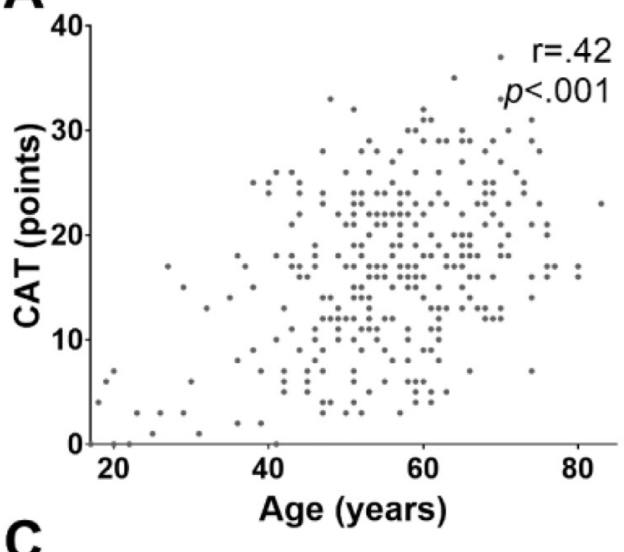

C

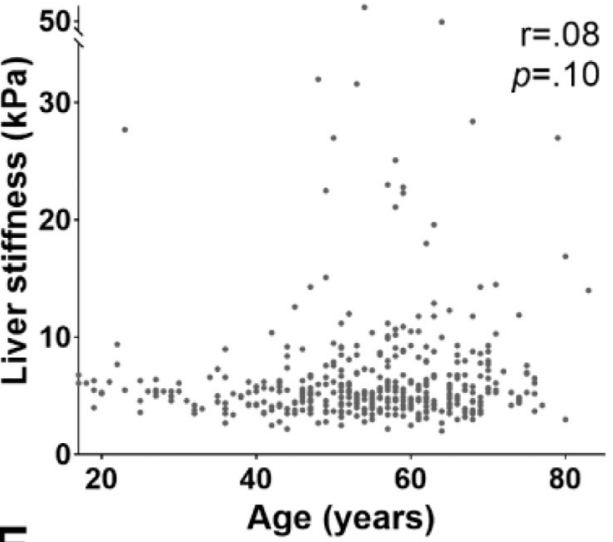

E

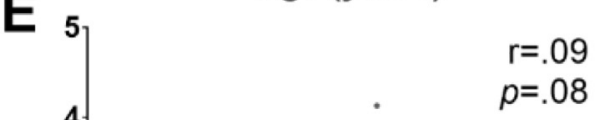

G

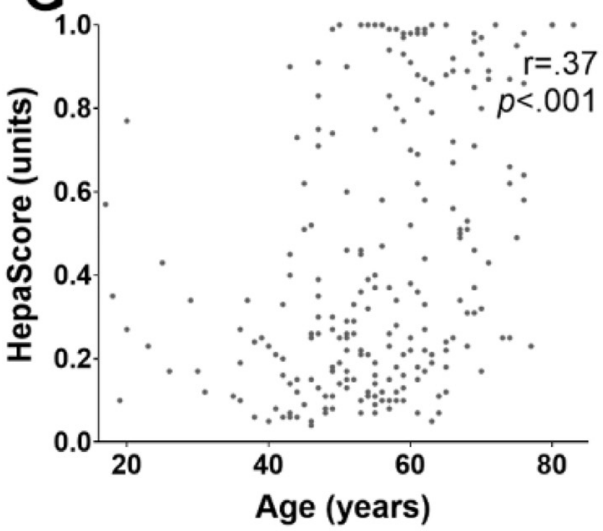

B
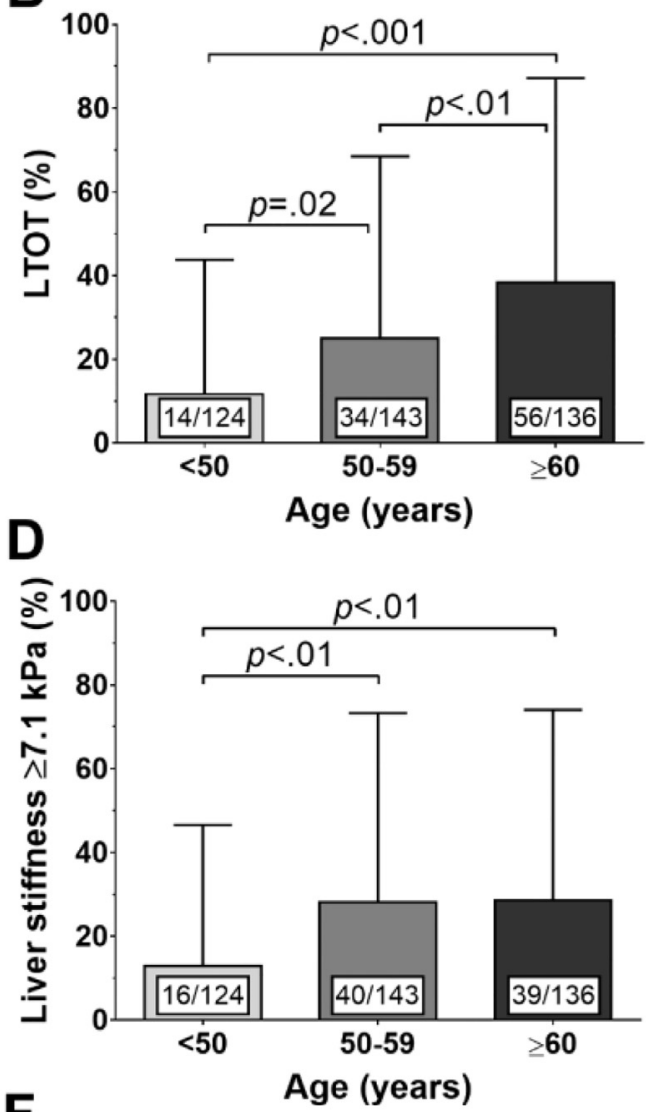

F

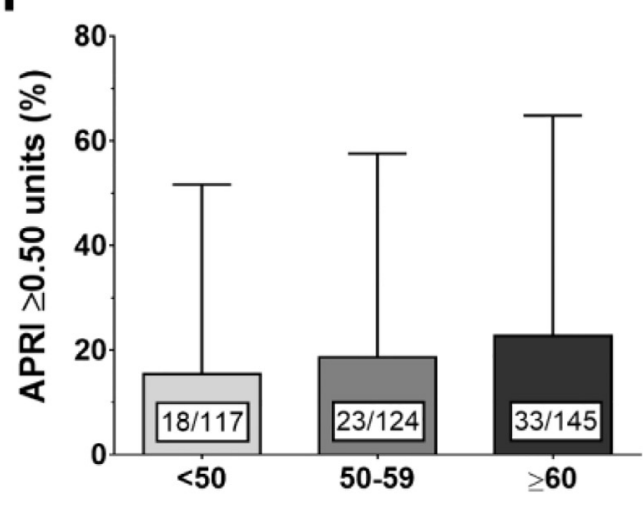

Age (years)

H

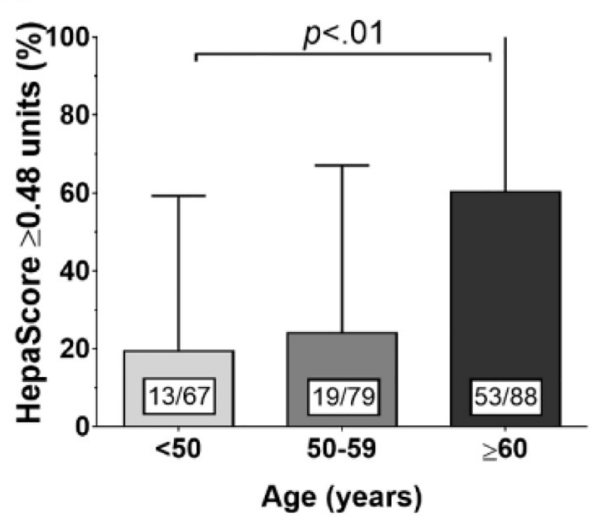


Supplementary Figure 2. Impact of age on lung and liver phenotype in carriers homozygous for the $A A T \mathrm{Pi}^{\star} \mathrm{Z}$ variant $\left(\mathrm{Pi}^{\star} \mathrm{ZZ}\right)$. Four hundred and three $\mathrm{Pi}^{\star} \mathrm{ZZ}$ carriers were analyzed by 3 independent, non-invasive liver fibrosis tests (TE [FibroScan], APRI, and HepaScore), while the lung function was estimated via the need for LTOT, as well as the CAT (a measure of lung functionrelated quality of life). (A) Linear correlation between age and CAT score. (B) Percentage of $\mathrm{Pi}^{\star} \mathrm{ZZ}$ carriers needing LTOT in the highlighted age groups. $(C, E, G)$ Linear correlation between age and non-invasive liver fibrosis parameters $(D, E, H)$ Percentage of $\mathrm{Pi}^{\star} \mathrm{ZZ}$ carriers with non-invasive test values suggesting the presence of significant liver fibrosis (LSM $\geq 7.1 \mathrm{kPa}$; APRI $\geq 0.50$ units; HepaScore $\geq 0.48$ units) in the depicted age groups. Notably, the analysis of the relation between HepaScore and age is confounded by the fact that age is a component of the HepaScore. 

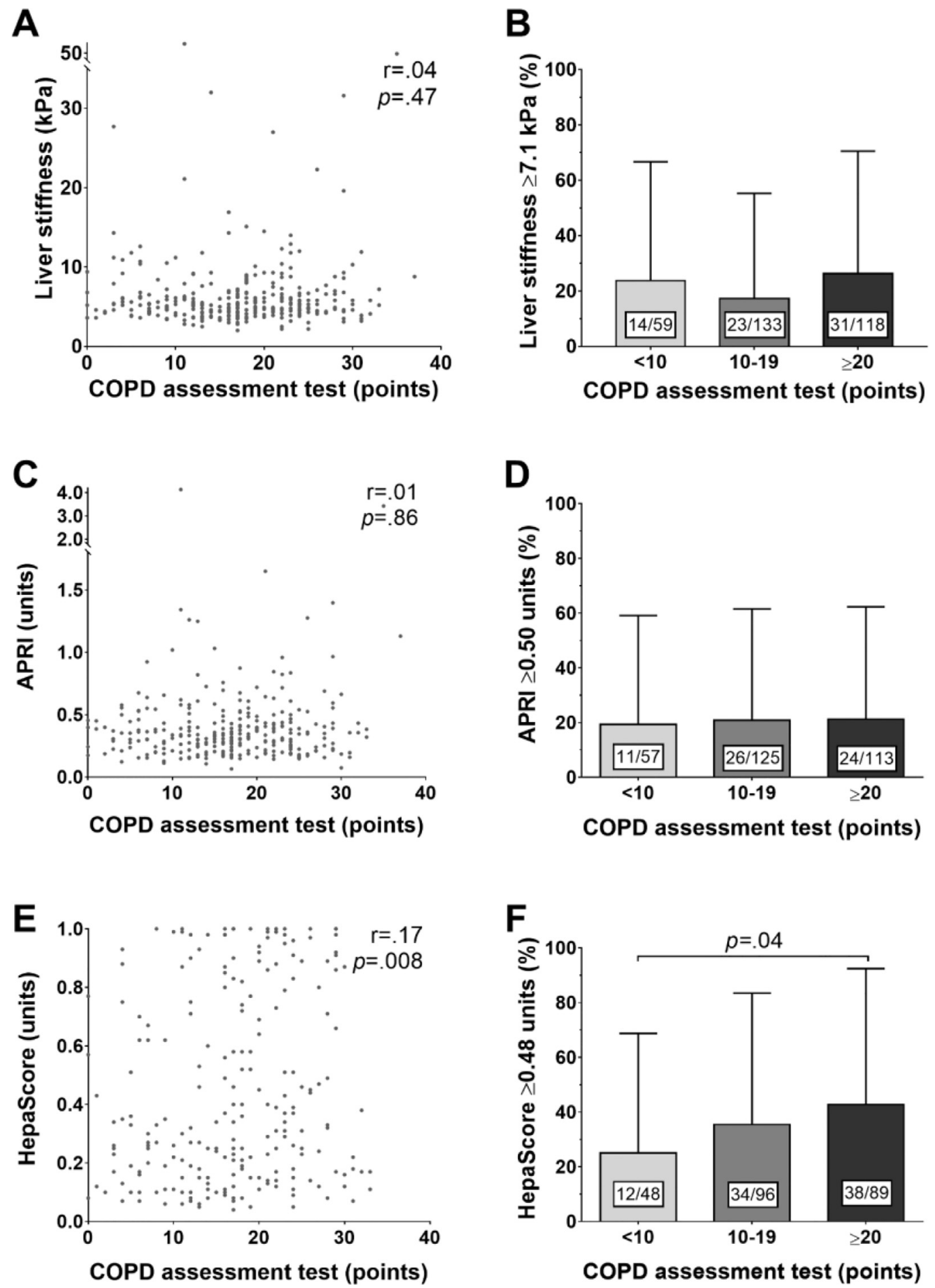

Supplementary Figure 3. Association of non-invasive fibrosis tests and COPD assessment test in carriers homozygous for the AAT $\mathrm{Pi}^{\star} \mathrm{Z}$ variant $\left(\mathrm{Pi}^{\star} \mathrm{ZZ}\right)$. Four hundred and three $\mathrm{Pi}^{\star} \mathrm{ZZ}$ carriers filled out standardized questionnaires and underwent noninvasive assessment of liver fibrosis via laboratory analysis and transient elastography. $(A, C, E)$ Linear correlation of $C A T$ score with liver stiffness, $A P R I$, and HepaScore values. $(B, D, F)$ Liver stiffness, APRI, and HepaScore values. in $\mathrm{Pi}^{\star} Z Z$ carriers with low, medium, and high CAT scores (CAT $<10,10-19$, and $\geq 20$, respectively) indicating the severity of chronic obstructive pulmonary disease-associated symptoms. 

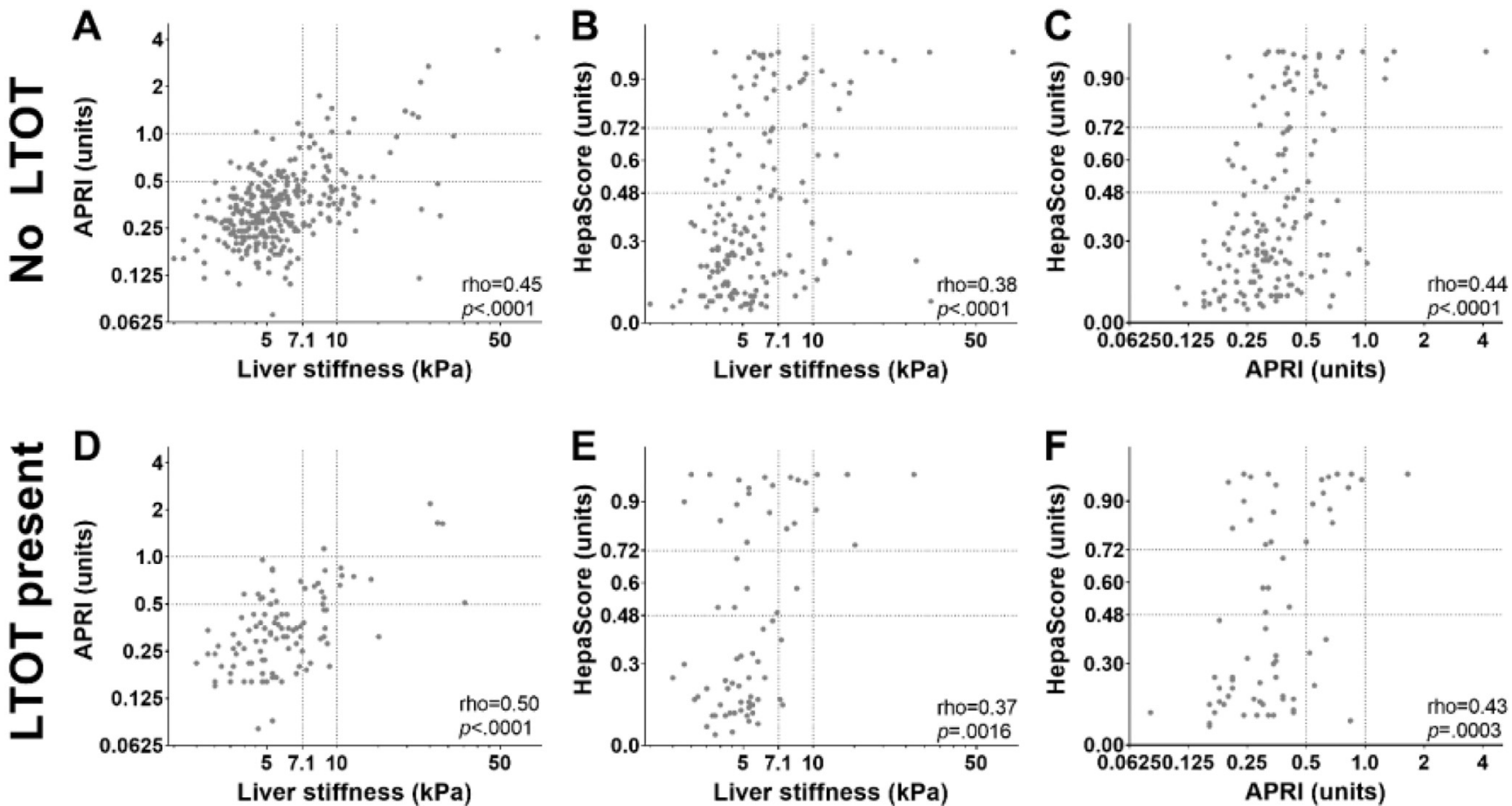

Supplementary Figure 4. Correlation of non-invasive liver fibrosis parameters in homozygous $\mathrm{Pi}^{\star} \mathrm{ZZ}$ carriers with and without LTOT. The dotted lines represent the proposed cutoffs for significant fibrosis (liver stiffness: $7.1 \mathrm{kPa}, \mathrm{APRI}$ : 0.50, HepaScore: 0.48 ) and advanced fibrosis (liver stiffness: $10 \mathrm{kPa}, \mathrm{APRI} 1.00$, HepaScore: 0.72$)$. $(A, D)$ Correlation of liver stiffness (log $\left.{ }_{10}\right)$ and APRI $\left(\log _{2}\right)$ in $\mathrm{Pi}^{\star} \mathrm{ZZ}$ carriers with and without LTOT. $(B, E)$ Correlation of liver stiffness $\left(\log _{10}\right)$ and HepaScore in Pi $Z Z$ carriers with and without LTOT. $(C, F)$ Correlation of APRI $\left(\log _{2}\right)$ and HepaScore in $\mathrm{Pi}^{\star} \mathrm{ZZ}$ carriers with and without LTOT. 

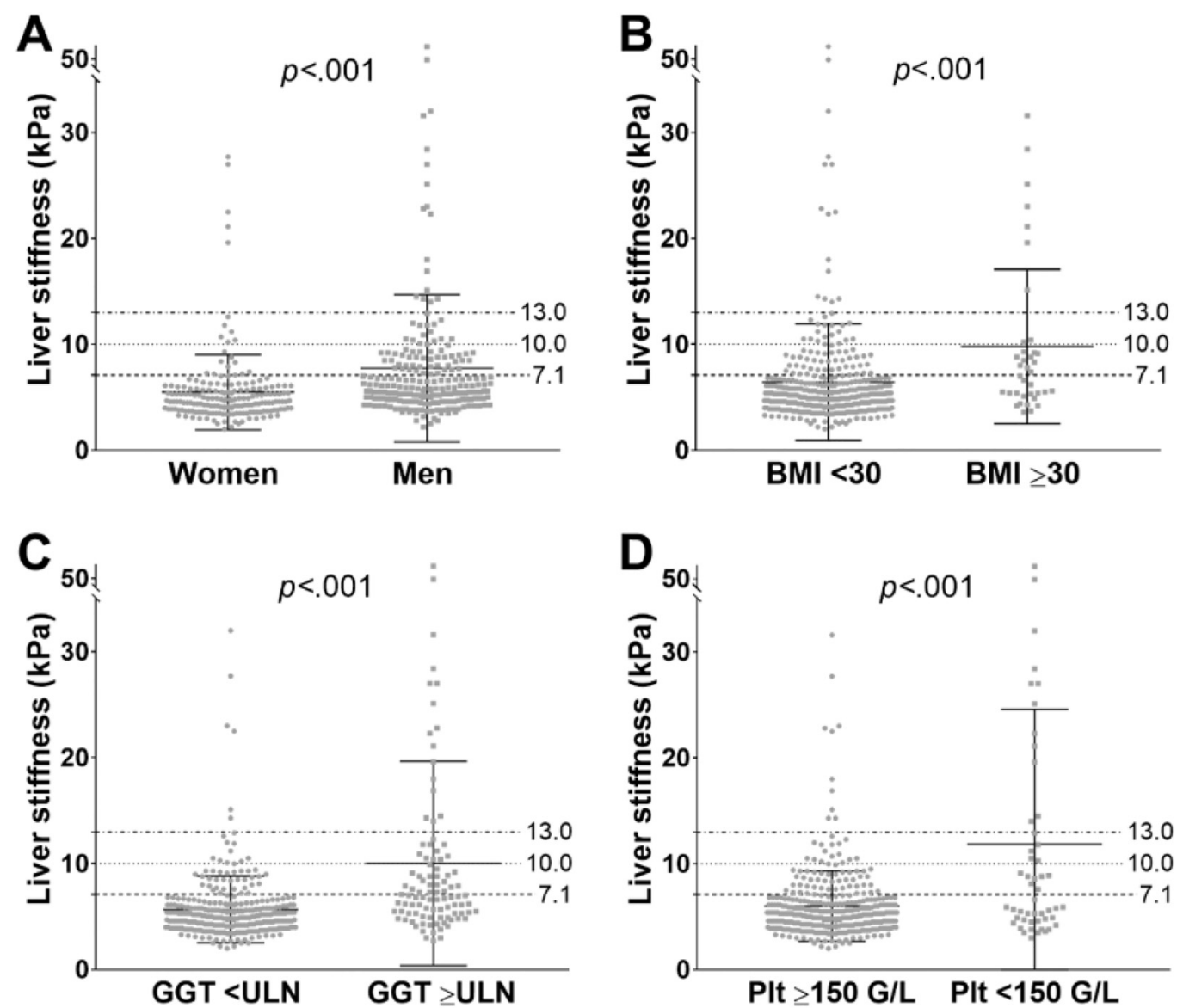

Supplementary Figure 5. Predictors of significant liver fibrosis as assessed by TE in homozygous carriers of the $A A T P{ }^{\star} Z$ variant $\left(\mathrm{Pi}^{\star} \mathrm{ZZ}\right)$. Four hundred and three $\mathrm{Pi}^{\star} \mathrm{ZZ}$ carriers were subjected to clinical evaluation, laboratory analysis, and noninvasive assessment by TE (FibroScan). $(A, B)$ Scatter plots of median liver stiffness determined by TE in women and men $(A)$ and in non-obese (body mass index $[\mathrm{BMI}]<30 \mathrm{~kg} / \mathrm{m}^{2}$ ) vs obese $\left(B M I \geq 30 \mathrm{~kg} / \mathrm{m}^{2}\right.$ ) individuals $(B)$. (C) Scatter plot of liver stiffness in $\mathrm{Pi}^{\star} \mathrm{ZZ}$ carriers with serum GGT activities within nomal reference range vs those with serum GGT activities above the sex-specific ULN. (D) Scatter plot of liver stiffness in $\mathrm{Pi}^{\star} \mathrm{ZZ}$ carriers with and without thrombocytopenia with a cutoff of $150 \mathrm{G} / \mathrm{L}$. The dotted lines represent the following etiology-unspecific cutoff levels: $7.1 \mathrm{kPa}$ (suggestive of fibrosis stage $\geq 2$ ), $10.0 \mathrm{kPa}$ (suggestive of fibrosis stage $\geq 3$ ), and $13.0 \mathrm{kPa}$ (suggestive of fibrosis stage 4). Plt, platelets. 

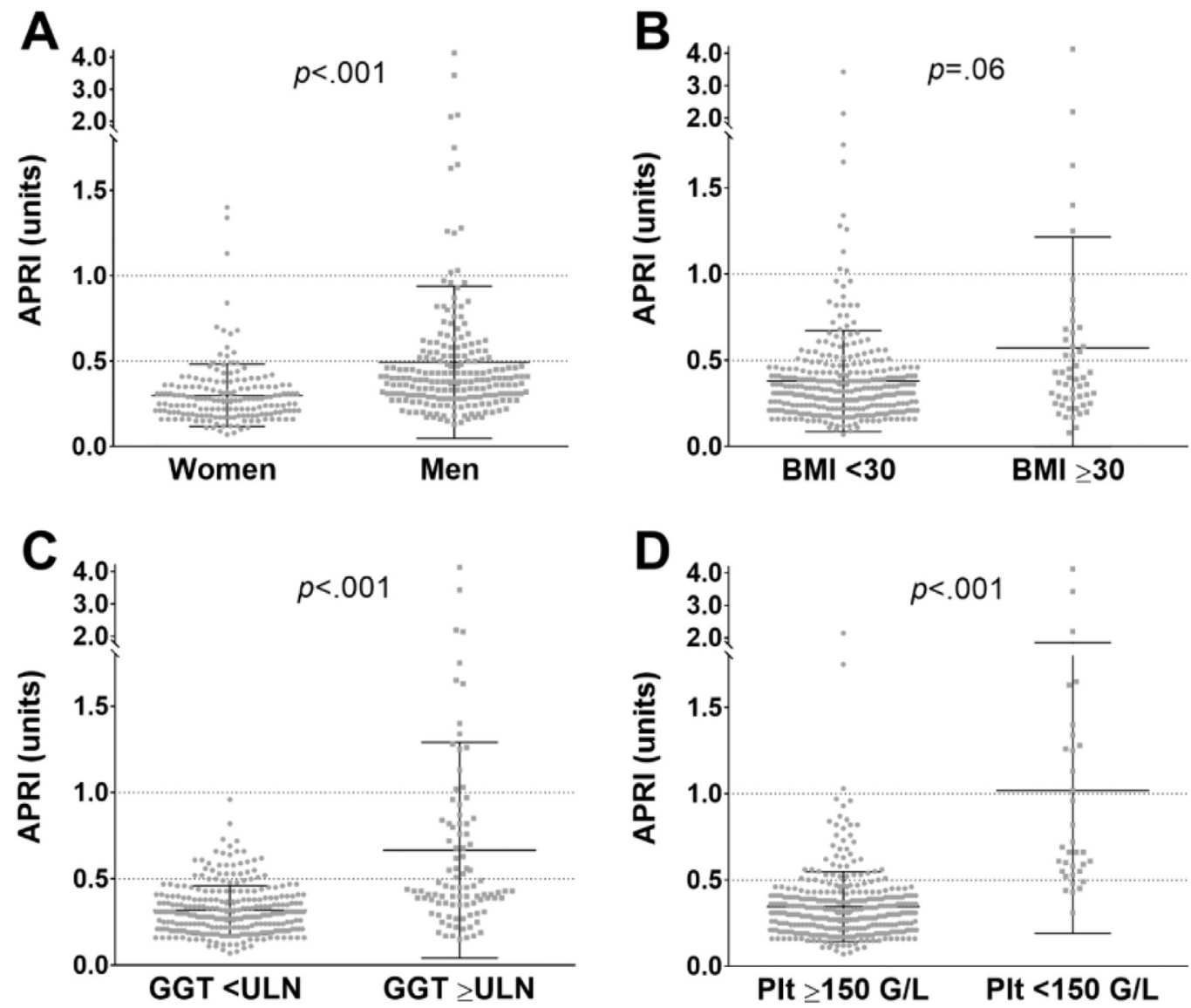

Supplementary Figure 6. Predictors of significant liver fibrosis as assessed by APRI in homozygous carriers of the AAT Pi* $Z$ variant $\left(\mathrm{Pi}^{\star} \mathrm{ZZ}\right)$. Four hundred and three $\mathrm{Pi}^{\star} \mathrm{ZZ}$ carriers and 234 non-carriers (exploratory cohort) were subjected to clinical evaluation and laboratory analysis. $(A, B)$ Scatter plots of APRI values in women and men $(A)$ and in non-obese (BMI $<30 \mathrm{~kg} /$ $\left.\mathrm{m}^{2}\right)$ vs obese $\left(B M I \geq 30 \mathrm{~kg} / \mathrm{m}^{2}\right)$ individuals. (C) Scatter plot of APRI levels in Pi`ZZ carriers with serum GGT activities within normal reference range vs those with serum GGT activities above the sex-specific ULN. (D) Scatter plot of APRI values in Pi ${ }^{\star} Z Z$ carriers with and without thrombocytopenia with a cutoff of $150 \mathrm{G} / \mathrm{L}$. The dotted lines represent the following etiologyunspecific cutoff levels: 0.5 (suggestive of fibrosis stage $\geq 2$ ) and 1.0 (suggestive of fibrosis stage $\geq 3$ ). BMI, body mass index; PIt, platelets. 

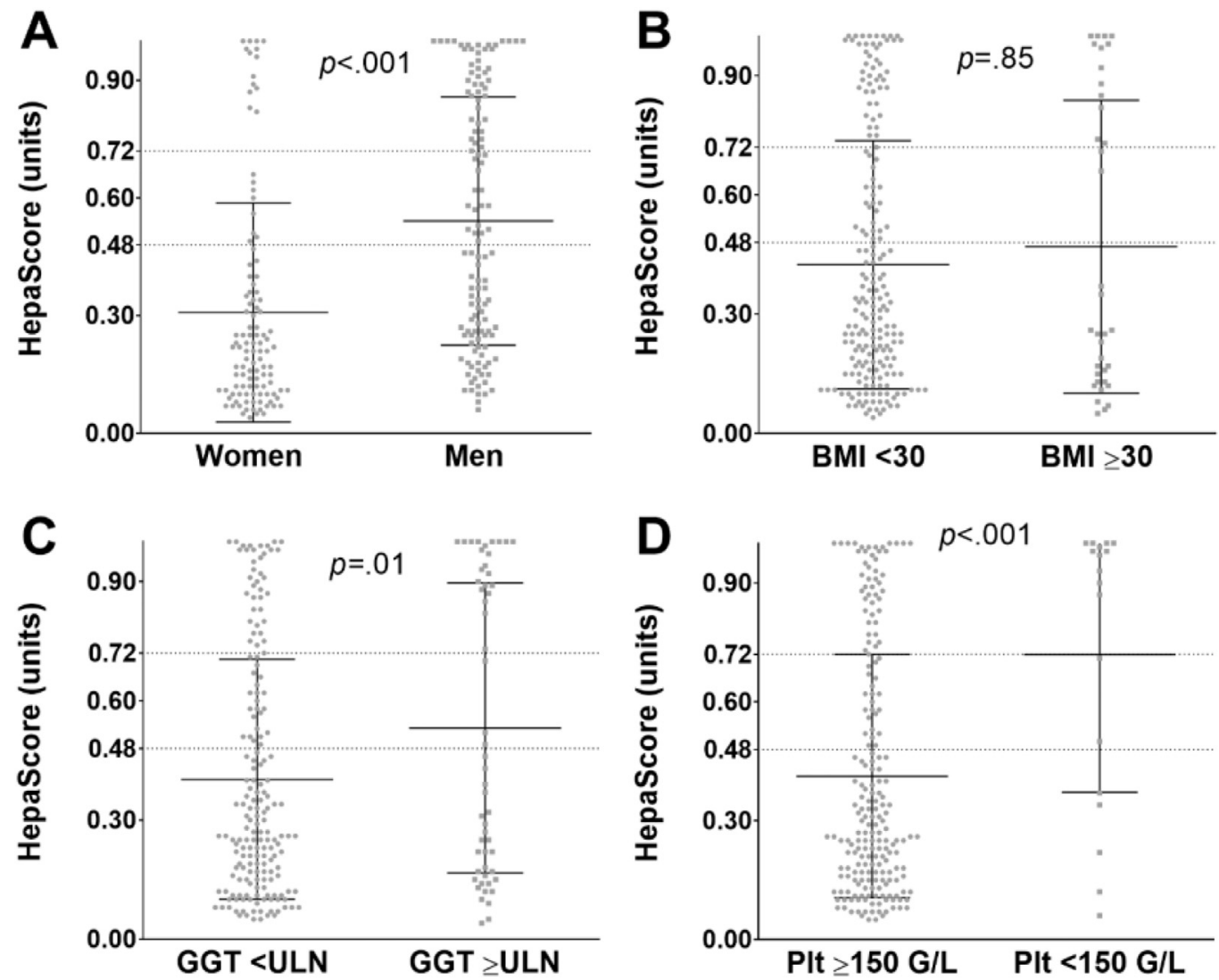

Supplementary Figure 7. Predictors of significant liver fibrosis as assessed by HepaScore in homozygous carriers of the AAT $\mathrm{Pi}^{\star} \mathrm{Z}$ variant $\left(\mathrm{Pi}^{\star} \mathrm{ZZ}\right)$. Four hundred and three $\mathrm{Pi}{ }^{\star} \mathrm{ZZ}$ carriers and 234 non-carriers (exploratory cohort) were subjected to clinical evaluation and laboratory analysis. $(A, B)$ Scatter plots of HepaScore values in women and men $(A)$ and in non-obese $(B M I<30$ $\mathrm{kg} / \mathrm{m}^{2}$ ) vs obese $\left(\mathrm{BMI} \geq 30 \mathrm{~kg} / \mathrm{m}^{2}\right.$ ) individuals. (C) Scatter plot of HepaScore levels in Pi^ZZ carriers with serum GGT activities within normal reference range vs those with serum GGT activities above the sex-specific ULN. (D) Scatter plot of HepaScore values in $\mathrm{Pi}^{\star} \mathrm{ZZ}$ carriers with and without thrombocytopenia with a cutoff of $150 \mathrm{G} / \mathrm{L}$. The dotted lines represent the following etiology-unspecific cutoff levels: 0.48 (suggestive of fibrosis stage $\geq 2$ ) and 0.72 (suggestive of fibrosis stage $\geq 3$ ). BMI, body mass index; Plt, platelets. 
Supplementary Table 1.Characteristics of Homozygous Carriers of the $\alpha 1$-Antitrypsin $\mathrm{Pi}^{\star} Z$ Variant (Pi^ZZ) From Participating Countries

\begin{tabular}{|c|c|c|c|c|c|}
\hline Variable & $\begin{array}{l}\text { Germany } \\
(n=254)\end{array}$ & $\begin{array}{l}\text { Austria } \\
(n=64)\end{array}$ & $\begin{array}{c}\text { Denmark } \\
(\mathrm{n}=26)\end{array}$ & $\begin{array}{l}\text { Portugal } \\
(\mathrm{n}=41)\end{array}$ & $\begin{array}{l}\text { Spain } \\
(n=18)\end{array}$ \\
\hline \multicolumn{6}{|l|}{ Characteristic } \\
\hline Age, $y$ & $54.8 \pm 11.9$ & $54.0 \pm 13.8$ & $58.4 \pm 11.1$ & $49.1 \pm 16.5$ & $48.6 \pm 15.9$ \\
\hline Women & 47 & 43 & 57 & 34 & 39 \\
\hline $\mathrm{BMI}, \mathrm{kg} / \mathrm{m}^{2}$ & $25.0 \pm 4.4$ & $24.1 \pm 4.3$ & $23.6 \pm 2.7$ & $25.4 \pm 5.7$ & $23.5 \pm 2.6$ \\
\hline Diabetes mellitus & 6 & 2 & 0 & 7 & 0 \\
\hline Mean alcohol consumption, $g / d$ & $6.3 \pm 9.3$ & $6.9 \pm 12.7$ & $12.4 \pm 14.2$ & $5.5 \pm 11.1$ & $4.1 \pm 6.9$ \\
\hline \multicolumn{6}{|l|}{ Liver fibrosis assessment } \\
\hline Liver stiffness, $k P a$ & $6.7 \pm 6.3$ & $5.9 \pm 3.0$ & $5.9 \pm 3.6$ & $8.2 \pm 6.6$ & $6.2 \pm 4.1$ \\
\hline Liver stiffness $\geq 7.1 \mathrm{kPa}$ & 23 & 23 & 15 & 39 & 6 \\
\hline Liver stiffness $\geq 10.0 \mathrm{kPa}$ & 14 & 8 & 8 & 24 & 6 \\
\hline APRI, units & $0.39 \pm 0.30$ & $0.45 \pm 0.55$ & $0.28 \pm 0.15$ & $0.47 \pm 0.44$ & $0.40 \pm 0.30$ \\
\hline $\mathrm{APRI} \geq 0.50$ units & 22.2 & 14.3 & 3.8 & 20.0 & 16.7 \\
\hline APRI $\geq 1.00$ units & 2.5 & 4.8 & 0 & 7.5 & 5.6 \\
\hline
\end{tabular}

NOTE. As patients from the Netherlands and Belgium were assessed in Germany, they are included in the category "Germany." Quantitative measures are expressed as mean \pm SD or as relative frequency (\%). Patients from exploratory cohort are shown.

BMI, body mass index.

Supplementary Table 2. Characteristics of Confirmatory Cohort Composed of Homozygous Carriers of the $\alpha 1$-Antitrypsin $\mathrm{Pi}^{\star} \mathrm{Z}$ Variant $\left(\mathrm{Pi}{ }^{\star} \mathrm{ZZ}\right)$

\begin{tabular}{|c|c|c|c|c|c|}
\hline Variable & $\begin{array}{l}\text { Germany } \\
(\mathrm{n}=71)\end{array}$ & $\begin{array}{l}\text { United Kingdom } \\
\qquad(\mathrm{n}=48)\end{array}$ & $\begin{array}{l}\text { Switzerland } \\
\quad(n=15)\end{array}$ & $\begin{array}{l}\text { Portugal } \\
(n=17)\end{array}$ & $\begin{array}{c}\text { Total } \\
(\mathrm{n}=151)\end{array}$ \\
\hline \multicolumn{6}{|l|}{ Characteristic } \\
\hline Women & 40.8 & 50.0 & $4 \overline{6} .7$ & 41.2 & $4 \overline{4} .4$ \\
\hline $\mathrm{BMI}, \mathrm{kg} / \mathrm{m}^{2}$ & $25.5 \pm 0.5$ & $26.6 \pm 0.7$ & $26.7 \pm 0.9$ & $24.1 \pm 0.9$ & $25.8 \pm 0.4$ \\
\hline Diabetes mellitus & $\overline{0}$ & $N \bar{A}$ & $\overline{0}$ & $5 . \overline{9}$ & NA \\
\hline Liver stiffness, $k P a$ & $7.0 \pm 0.8$ & $6.5 \pm 0.4$ & $6.0 \pm 0.4$ & $6.8 \pm 0.7$ & $6.7 \pm 0.4$ \\
\hline Liver stiffness $\geq 7.1 \mathrm{kPa}$ & 22.5 & 27.1 & 33.3 & 41.2 & 27.2 \\
\hline Liver stiffness $\geq 10.0 \mathrm{kPa}$ & 12.7 & 16.7 & 6.7 & 11.8 & 13.2 \\
\hline APRI, units & $0.46 \pm 0.52$ & NA & $0.38 \pm 0.20$ & NA & $0.45 \pm 0.48$ \\
\hline APRI $\geq 0.50$ units & 4.4 & - & 20 & - & 5.4 \\
\hline APRI $\geq 1.00$ units & 0 & - & 0 & - & 0 \\
\hline
\end{tabular}

NOTE. Quantitative measures are expressed as mean \pm SD or as relative frequency (\%).

BMI, body mass index; NA, not available. 
Supplementary Table 3.Selected Laboratory Values in Homozygous Carriers of the $\alpha 1$-Antitrypsin $\mathrm{Pi}^{\star} \mathrm{Z}$ Variant $\left(\mathrm{Pi}^{\star} \mathrm{ZZ}\right)$ and $\mathrm{Pi}^{\star} Z$ Non-Carriers

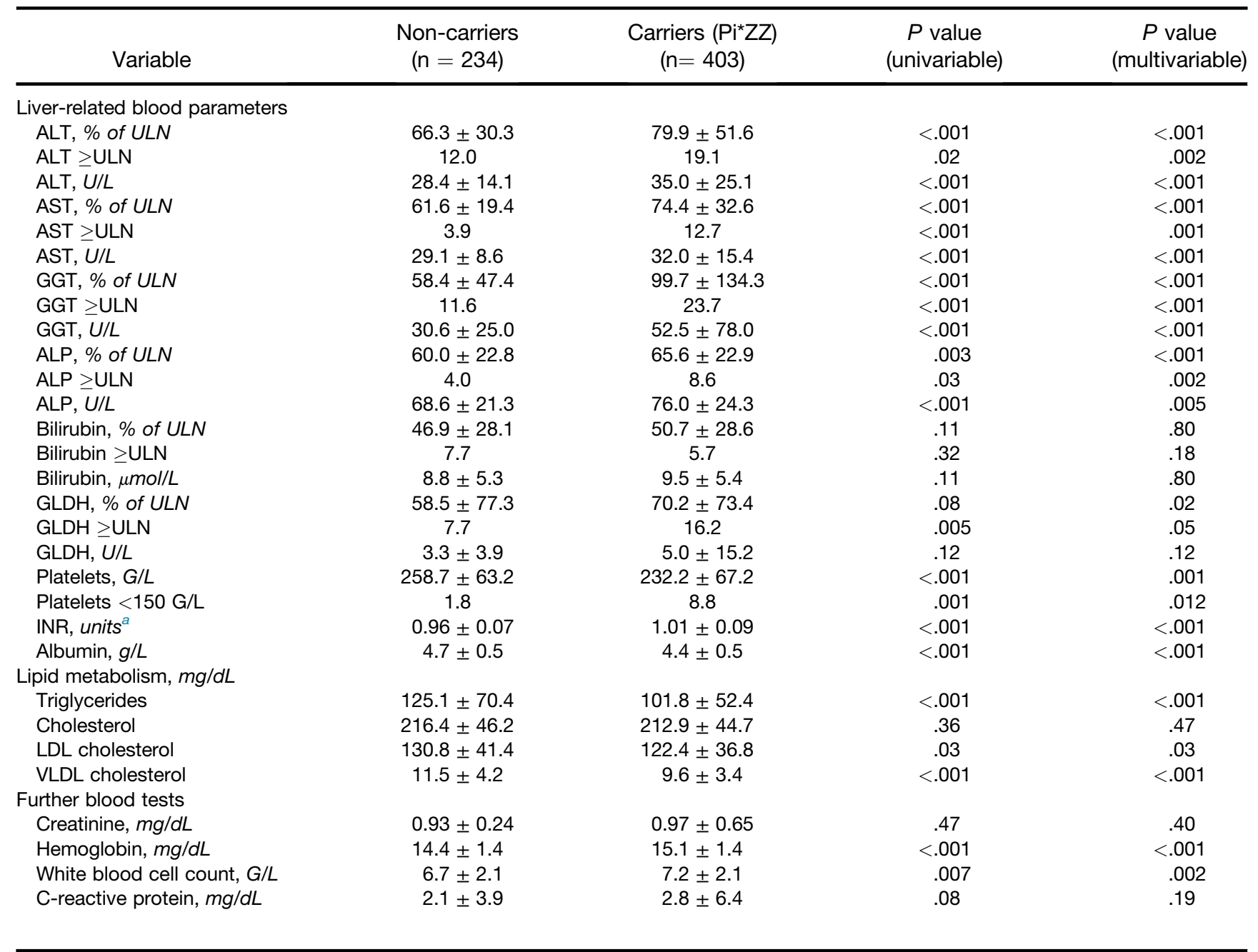

NOTE. Quantitative measures are expressed as mean \pm SD or as relative frequency (\%). Multivariable analyses were adjusted for age, sex, BMI, presence of diabetes mellitus, and mean alcohol consumption.

ALP, alkaline phosphatase; GLDH, glutamate dehydrogenase; INR, international normalized ratio; LDL, low-density lipoprotein; LLN, sex-specific lower limit of normal; ULN, sex-specific upper limit of normal; VLDL, very-low-density lipoprotein.

${ }^{a}$ Only patients not taking anticoagulant medication were analyzed. 
Supplementary Table 4. Characteristics of Homozygous Carriers of the $\alpha 1$-Antitrypsin $\mathrm{Pi}^{\star} \mathrm{Z}$ Variant $\left(\mathrm{Pi}^{\star} \mathrm{ZZ}\right)$ Who Were Recruited in Germany vs Those Who Were Recruited in Other European Countries

\begin{tabular}{|c|c|c|c|}
\hline Variable & $\begin{array}{c}\text { German } \mathrm{Pi}^{\star} \mathrm{ZZ} \\
\text { carriers }(\mathrm{n}=381)\end{array}$ & $\begin{array}{l}\text { Non-German } \mathrm{Pi}^{\star} \mathrm{ZZ} \\
\text { carriers }(\mathrm{n}=173)\end{array}$ & $\begin{array}{c}P \\
\text { value }\end{array}$ \\
\hline \multicolumn{4}{|l|}{ Characteristics } \\
\hline Age, $y$ & $55.6 \pm 12.3$ & $51.2 \pm 14.4$ & $<.001$ \\
\hline Women & 45.9 & 43.4 & .57 \\
\hline $\mathrm{BMI}, \mathrm{kg} / \mathrm{m}^{2}$ & $25.0 \pm 4.4$ & $25.2 \pm 4.6$ & .50 \\
\hline Mean alcohol consumption, $g / d$ & $5.6 \pm 9.4$ & $5.2 \pm 9.9$ & .72 \\
\hline \multicolumn{4}{|l|}{ Risk factors } \\
\hline $\mathrm{BMI} \geq 30 \mathrm{~kg} / \mathrm{m}^{2}$ & 15.7 & 13.8 & .55 \\
\hline Waist circumference, $\mathrm{cm}$ & $95.1 \pm 14.4$ & $95.3 \pm 11.7$ & .92 \\
\hline Diabetes mellitus & 4.5 & 2.8 & .44 \\
\hline \multicolumn{4}{|l|}{ Lung status } \\
\hline Cigarette consumption, pack-years & $10.6 \pm 12.6$ & $11.2 \pm 17.7$ & .72 \\
\hline CAT score, points & $17.2 \pm 8.2$ & $14.2 \pm 8.0$ & .03 \\
\hline LTOT & 28.0 & 13.9 & .003 \\
\hline \multicolumn{4}{|l|}{ Liver status } \\
\hline Liver stiffness, $k P a$ & $6.6 \pm 6.0$ & $6.9 \pm 4.4$ & .60 \\
\hline Liver stiffness $\geq 7.1 \mathrm{kPa}$ & 22.3 & 29.5 & .07 \\
\hline Liver stiffness $\geq 10.0 \mathrm{kPa}$ & 12.3 & 16.2 & .22 \\
\hline $\mathrm{CAP}, d B / m$ & $268 \pm 58$ & $262 \pm 48$ & .37 \\
\hline $\mathrm{CAP} \geq 248 \mathrm{~dB} / \mathrm{m}$ & 62.2 & 62.4 & .98 \\
\hline $\mathrm{CAP} \geq 280 \mathrm{~dB} / \mathrm{m}$ & 38.4 & 35.3 & .60 \\
\hline APRI, units & $0.41 \pm 0.39$ & $0.42 \pm 0.36$ & .81 \\
\hline APRI $\geq 0.50$ units & 20.1 & 17.9 & .62 \\
\hline APRI $\geq 1.00$ units & 4.1 & 5.7 & .50 \\
\hline HepaScore, units & $0.43 \pm 0.32$ & $0.41 \pm 0.30$ & .85 \\
\hline HepaScore $\geq 0.48$ units & 36.4 & 33.3 & .85 \\
\hline HepaScore $\geq 0.72$ units & 26.2 & 11.1 & .31 \\
\hline
\end{tabular}

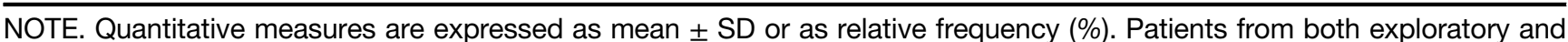
confirmatory cohort were included.

$\mathrm{BMI}$, body mass index. 
Supplementary Table 5. Characteristics of $\mathrm{Pi}^{\star} \mathrm{Z}$ Non-Carriers and Homozygous Carriers of the $\alpha 1$-Antitrypsin $\mathrm{Pi}$ Z $\mathrm{V}$ Variant (PiZZ) Not Receiving $\alpha 1$-Antitrypsin Augmentation Therapy

\begin{tabular}{|c|c|c|c|c|}
\hline Variable & $\begin{array}{c}\text { Non-carriers } \\
(n=234)\end{array}$ & $\begin{array}{c}\mathrm{Pi}^{\star} \mathrm{ZZ} \text { (non-augmented) } \\
(\mathrm{n}=168)\end{array}$ & $\begin{array}{c}P \text { value } \\
\text { (univariable) }\end{array}$ & $\begin{array}{c}P \text { value } \\
\text { (multivariable) }\end{array}$ \\
\hline \multicolumn{5}{|l|}{ Characteristics } \\
\hline Age, $y$ & $53.1 \pm 14.6$ & $49.1 \pm 15.2$ & .03 & - \\
\hline Women & 48.7 & 50.6 & .71 & - \\
\hline $\mathrm{BMI}, \mathrm{kg} / \mathrm{m}^{2}$ & $25.1 \pm 3.8$ & $24.3 \pm 3.8$ & .05 & - \\
\hline $\mathrm{BMI} \geq 30 \mathrm{~kg} / \mathrm{m}^{2}$ & 14.9 & 10.8 & .27 & - \\
\hline Diabetes mellitus & 5.6 & 3.9 & .48 & - \\
\hline Mean alcohol consumption, $g / d$ & $7.9 \pm 10.1$ & $5.8 \pm 10.4$ & 0.06 & - \\
\hline \multicolumn{5}{|l|}{ AATD-related features } \\
\hline AAT serum level, $m g / d L$ & $139.7 \pm 25.3$ & $28.6 \pm 16.6$ & $<.001$ & $<.001$ \\
\hline CAT score, points & $6.8 \pm 6.0$ & $14.2 \pm 8.3$ & $<.001$ & $<.001$ \\
\hline LTOT & 0.4 & 11.3 & $<.001$ & $<.001$ \\
\hline \multicolumn{5}{|l|}{ Liver status } \\
\hline Liver stiffness, $\mathrm{kPa}$ & $4.6 \pm 1.7$ & $7.0 \pm 6.3$ & $<.001$ & $<.001$ \\
\hline Liver stiffness $\geq 7.1 \mathrm{kPa}$ & 6.6 & 23.8 & $<.001$ & $<.001$ \\
\hline $\mathrm{CAP}, d B / m$ & $246.2 \pm 59.3$ & $260.7 \pm 54.9$ & .02 & $<.001$ \\
\hline APRI, units & $0.27 \pm 0.12$ & $0.42 \pm 0.40$ & $<.001$ & $<.001$ \\
\hline HepaScore, units & $0.25 \pm 0.21$ & $0.41 \pm 0.32$ & $<.001$ & $<.001$ \\
\hline ALT, $\%$ of ULN & $66.2 \pm 30.1$ & $76.6 \pm 52.8$ & .01 & .001 \\
\hline AST, $\%$ of ULN & $61.6 \pm 19.4$ & $76.2 \pm 31.4$ & $<.001$ & $<.001$ \\
\hline GGT, $\%$ of ULN & $58.4 \pm 47.4$ & $58.4 \pm 47.4$ & $<.001$ & $<.001$ \\
\hline ALP, $\%$ of ULN & $60.0 \pm 22.8$ & $98.9 \pm 115.7$ & .004 & $<.001$ \\
\hline Bilirubin, \% of ULN & $46.9 \pm 28.1$ & $56.2 \pm 34.5$ & .003 & .003 \\
\hline Platelets, $G / L$ & $258.7 \pm 63.2$ & $229.6 \pm 71.4$ & $<.001$ & $<.001$ \\
\hline INR, units ${ }^{a}$ & $0.96 \pm 0.07$ & $1.01 \pm 0.10$ & $<.001$ & $<.001$ \\
\hline Albumin, $g / L$ & $4.7 \pm 0.5$ & $4.5 \pm 0.5$ & $<.001$ & $<.001$ \\
\hline \multicolumn{5}{|l|}{ Lipid metabolism } \\
\hline Triglycerides, $\mathrm{mg} / \mathrm{dL}$ & $125.1 \pm 70.4$ & $100.1 \pm 49.8$ & $<.001$ & .002 \\
\hline VLDL cholesterol, $m g / d L$ & $11.5 \pm 4.2$ & $9.4 \pm 3.3$ & $<.001$ & .001 \\
\hline
\end{tabular}

NOTE. Quantitative measures are expressed as mean \pm SD or as relative frequency (\%). Multivariable analyses were adjusted for age, sex, BMI, presence of diabetes mellitus, and mean alcohol consumption.

BMI, body mass index; INR, international normalized ratio; LDL, low-density lipoprotein; LLN, sex-specific lower limit of normal; ULN, sex-specific upper limit of normal; VLDL, very-low-density lipoprotein.

${ }^{a}$ Only patients not taking anticoagulant medication were considered. 
Supplementary Table 6. Characteristics of Homozygous Carriers of the $\alpha 1$-Antitrypsin $\mathrm{Pi}^{\star} \mathrm{Z}$ Variant (Pi^ZZ) Based on Their Age

\begin{tabular}{|c|c|c|c|c|c|c|c|}
\hline \multirow[b]{2}{*}{ Variable } & \multicolumn{3}{|c|}{$\mathrm{Pi}^{\star} \mathrm{ZZ}$} & \multicolumn{4}{|c|}{$P$ value } \\
\hline & $\begin{array}{c}<50 y \\
(n=124)\end{array}$ & $\begin{array}{c}50-59 y \\
(n=143)\end{array}$ & $\begin{array}{c}\geq 60 y \\
(n=136)\end{array}$ & Overall & $<50$ vs $50-59$ & $<50$ vs $\geq 60$ & $50-59$ vs $\geq 60$ \\
\hline \multicolumn{8}{|l|}{ Characteristics } \\
\hline Age, $y$ & $38.5 \pm 9.3$ & $55.2 \pm 3.1$ & $67.1 \pm 4.9$ & $<.001$ & $<.001$ & $<.001$ & $<.001$ \\
\hline Women & 43.5 & 44.8 & 47.8 & .78 & .84 & .49 & .61 \\
\hline $\mathrm{BMI}, \mathrm{kg} / \mathrm{m}^{2}$ & $24.7 \pm 4.3$ & $24.8 \pm 4.4$ & $24.7 \pm 4.4$ & .90 & .94 & .69 & .72 \\
\hline Mean alcohol, g/d & $5.1 \pm 7.9$ & $7.6 \pm 10.9$ & $6.7 \pm 11.3$ & .09 & .05 & .88 & .08 \\
\hline AAT serum level, ${ }^{a} \mathrm{mg} / \mathrm{dL}$ & $25.7 \pm 11.9$ & $29.6 \pm 22.5$ & $31.6 \pm 12.2$ & .01 & .42 & .002 & .02 \\
\hline \multicolumn{8}{|l|}{ Risk factors } \\
\hline $\mathrm{BMI} \geq 30 \mathrm{~kg} / \mathrm{m}^{2}$ & 14.5 & 12.1 & 12.5 & .82 & .56 & .64 & .91 \\
\hline Diabetes mellitus & 3.7 & 6.2 & 5.0 & .70 & .40 & .65 & .68 \\
\hline Relevant alcohol intake ${ }^{b}$ & 8.1 & 13.8 & 8.8 & .27 & .16 & .85 & .21 \\
\hline \multicolumn{8}{|l|}{ Lung status } \\
\hline Smoking, pack-years & $9.2 \pm 13.1$ & $12.6 \pm 15.2$ & $8.0 \pm 12.8$ & .01 & .07 & .27 & .01 \\
\hline CAT score, points & $13.0 \pm 8.1$ & $17.2 \pm 7.1$ & $19.7 \pm 6.9$ & $<.001$ & $<.001$ & $<.001$ & .02 \\
\hline LTOT & 11.6 & 23.5 & 41.3 & $<.001$ & .02 & $<.001$ & .002 \\
\hline AAT substitution & 48.4 & 57.0 & 68.4 & .01 & .16 & .001 & .05 \\
\hline \multicolumn{8}{|l|}{ Liver status } \\
\hline Liver stiffness, $\mathrm{kPa}$ & $5.9 \pm 4.0$ & $7.2 \pm 7.2$ & $6.9 \pm 5.4$ & .11 & .11 & .04 & .74 \\
\hline $\mathrm{LSM} \geq 7.1 \mathrm{kPa}$ & 12.9 & 28.0 & 28.7 & .004 & .003 & .002 & .90 \\
\hline $\mathrm{LSM} \geq 10.0 \mathrm{kPa}$ & 7.3 & 16.8 & 16.2 & .05 & .02 & .03 & .90 \\
\hline $\mathrm{CAP}, d B / m$ & $258.0 \pm 56.3$ & $267.5 \pm 58.1$ & $273.0 \pm 52.6$ & .11 & .19 & .04 & .37 \\
\hline $\mathrm{CAP} \geq 280 \mathrm{~dB} / \mathrm{m}$ & 33.7 & 37.4 & 44.3 & .25 & .55 & .11 & .27 \\
\hline APRI, units & $0.34 \pm 0.20$ & $0.44 \pm 0.48$ & $0.43 \pm 0.35$ & .02 & .03 & .02 & 82 \\
\hline HepaScore, units & $0.30 \pm 0.25$ & $0.38 \pm 0.32$ & $0.58 \pm 0.31$ & .006 & .29 & $<.001$ & $<.001$ \\
\hline ALT, \% of ULN & $78.8 \pm 56.4$ & $84.6 \pm 57.1$ & $75.9 \pm 39.4$ & .51 & .22 & .73 & .49 \\
\hline AST, $\%$ of ULN & $65.4 \pm 26.6$ & $79.0 \pm 39.4$ & $77.2 \pm 28.4$ & $<.001$ & $<.001$ & $<.001$ & .72 \\
\hline GGT, $\%$ of ULN & $68.3 \pm 40.9$ & $109.7 \pm 148.1$ & $117.6 \pm 166.6$ & .01 & .01 & .003 & .58 \\
\hline ALP, $\%$ of ULN & $58.9 \pm 17.7$ & $67.2 \pm 25.3$ & $70.0 \pm 23.1$ & $<.001$ & .004 & $<.001$ & .18 \\
\hline Bilirubin, \% of ULN & $52.6 \pm 31.3$ & $50.8 \pm 30.0$ & $53.2 \pm 25.2$ & .37 & .57 & .47 & .17 \\
\hline Platelets, $G / L$ & $234.4 \pm 56.1$ & $239.8 \pm 70.9$ & $222.1 \pm 71.3$ & .08 & .63 & .10 & .04 \\
\hline INR, units ${ }^{c}$ & $1.01 \pm 0.09$ & $1.02 \pm 0.09$ & $1.02 \pm 0.10$ & .45 & .52 & .21 & .52 \\
\hline Albumin, $g / L$ & $4.5 \pm 0.5$ & $4.5 \pm 0.5$ & $4.3 \pm 0.5$ & $<.001$ & .23 & $<.001$ & .003 \\
\hline \multicolumn{8}{|l|}{ Lipid metabolism, mg/dL } \\
\hline Triglycerides & $93.7 \pm 41.8$ & $104.3 \pm 57.6$ & $106.5 \pm 54.6$ & .15 & .15 & .07 & .61 \\
\hline Cholesterol & $197.5 \pm 35.4$ & $219.6 \pm 38.9$ & $219.8 \pm 52.2$ & $<.001$ & $<.001$ & $<.001$ & .48 \\
\hline LDL cholesterol & $112.0 \pm 32.6$ & $128.7 \pm 36.6$ & $125.2 \pm 38.7$ & $<.001$ & $<.001$ & .01 & .20 \\
\hline VLDL cholesterol & $9.2 \pm 3.3$ & $10.1 \pm 3.5$ & $9.4 \pm 3.3$ & .45 & .26 & .88 & .39 \\
\hline
\end{tabular}

NOTE. Quantitative measures are expressed as mean \pm SD or as relative frequency (\%).

ALP, alkaline phosphatase; BMI, body mass index; INR, international normalized ratio; LDL, low-density lipoprotein; LLN, sexspecific lower limit of normal; ULN, sex-specific upper limit of normal; VLDL, very-low-density lipoprotein.

${ }^{a} \mathrm{AAT}$ serum levels of $\mathrm{Pi}^{\star} \mathrm{Z}$ non-carriers and $\mathrm{Pi}^{\star} \mathrm{ZZ}$ subjects, who did not receive AAT augmentation therapy, are shown. Mean AAT serum level in all $\mathrm{Pi}^{\star} \mathrm{ZZ}$ patients were $62.6 \pm 53.0 \mathrm{mg} / \mathrm{dL}(<50 \mathrm{y}) \mathrm{vs} 72.4 \pm 50.4 \mathrm{mg} / \mathrm{dL}(50-59 \mathrm{y}) \mathrm{vs} 81.9 \pm 53.3 \mathrm{mg} / \mathrm{dL}$ $(\geq 60 \mathrm{y})$.

${ }^{b}$ Alcohol intake $>12 \mathrm{~g} / \mathrm{d}$ for women and $>24 \mathrm{~g} / \mathrm{d}$ for men (individuals with alcohol consumption $>40 \mathrm{~g} / \mathrm{d}$ for females or $>60 \mathrm{~g} /$ $\mathrm{d}$ for males had been excluded a priori).

${ }^{c}$ Only patients not taking anticoagulant medication were considered. 
Supplementary Table 7.Characteristics of Homozygous Carriers of the $\alpha 1$-Antitrypsin $\mathrm{Pi}^{\star} \mathrm{Z}$ Variant (Pi^ZZ) Based on Their Sex

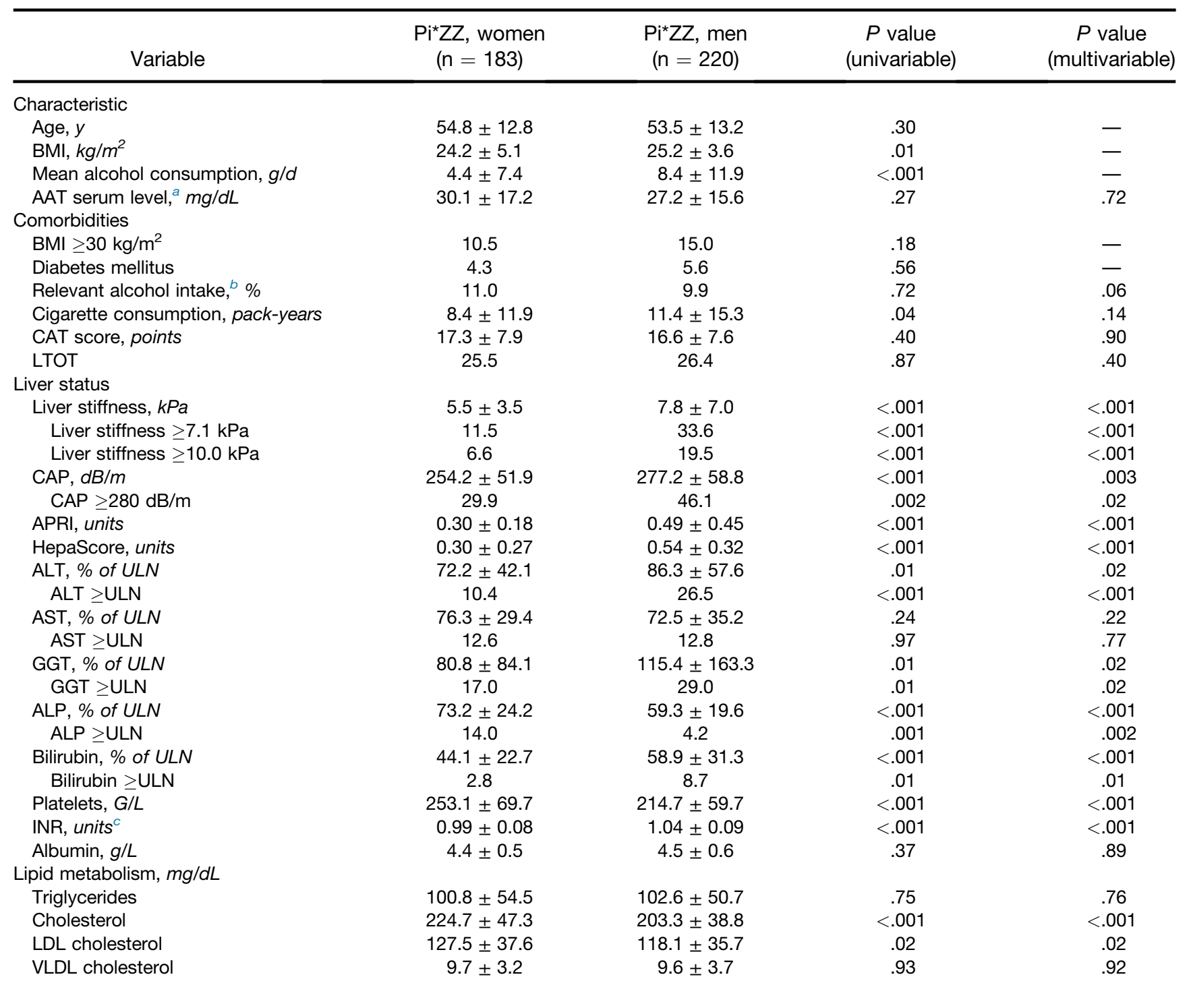

NOTE. Quantitative measures are expressed as mean \pm SD or as relative frequency (\%). Multivariable analyses were adjusted for age, BMI, presence of diabetes mellitus, and mean alcohol consumption.

ALP, alkaline phosphatase; BMI, body mass index; INR, international normalized ratio; LDL, low-density lipoprotein; LLN, sexspecific lower limit of normal; ULN, sex-specific upper limit of normal; VLDL, very-low-density lipoprotein.

${ }^{a}$ AAT serum levels of $\mathrm{Pi}^{\star} \mathrm{Z}$ non-carriers and $\mathrm{Pi}^{\star} \mathrm{ZZ}$ subjects, who did not receive AAT augmentation therapy are shown. Mean AAT serum level in $\mathrm{Pi}^{\star} \mathrm{ZZ}$ women was $70.2 \pm 49.9 \mathrm{mg} / \mathrm{dL}$ and in $\mathrm{Pi}^{\star} \mathrm{ZZ}$ men $74.4 \pm 54.85 \mathrm{mg} / \mathrm{dL}$.

${ }^{b}$ Alcohol intake $>12 \mathrm{~g} / \mathrm{d}$ for women and $>24 \mathrm{~g} / \mathrm{d}$ for men (individuals with alcohol consumption $>40 \mathrm{~g} / \mathrm{d}$ for females or $>60 \mathrm{~g} /$ $\mathrm{d}$ for males had been excluded a priori).

${ }^{c}$ Only patients not taking anticoagulant medication were considered. 
Supplementary Table 8. Characteristics of Homozygous Carriers of the $\alpha 1$-Antitrypsin $\mathrm{Pi}^{\star} \mathrm{Z}$ Variant (Pi^ $\left.\mathrm{ZZ}\right)$ With and Without a Liver Stiffness Measurement Suggesting Significant Liver Fibrosis

\begin{tabular}{|c|c|c|c|}
\hline Variable & $\begin{array}{c}\mathrm{Pi}^{\star} \mathrm{ZZ}, \mathrm{LSM}<7.1 \mathrm{kPa} \\
(\mathrm{n}=308)\end{array}$ & $\begin{array}{c}\mathrm{Pi}^{\star} Z Z, \quad L S M \geq 7.1 \mathrm{kPa} \\
(\mathrm{n}=95)\end{array}$ & $\begin{array}{c}P \text { value } \\
\text { (univariable) }\end{array}$ \\
\hline \multicolumn{4}{|l|}{ Characteristics } \\
\hline Age, $y$ & $52.9 \pm 13.4$ & $57.8 \pm 11.3$ & .001 \\
\hline Women & 52.6 & 22.1 & $<.001$ \\
\hline $\mathrm{BMI}, \mathrm{kg} / \mathrm{m}^{2}$ & $24.2 \pm 3.7$ & $26.6 \pm 5.7$ & $<.001$ \\
\hline Mean alcohol consumption, $g / d$ & $6.5 \pm 10.0$ & $7.0 \pm 11.3$ & .71 \\
\hline AAT serum level, ${ }^{a} \mathrm{mg} / \mathrm{dL}$ & $30.9 \pm 18.0$ & $27.9 \pm 16.1$ & .98 \\
\hline \multicolumn{4}{|l|}{ Comorbidities } \\
\hline $\mathrm{BMI} \geq 30 \mathrm{~kg} / \mathrm{m}^{2}$ & 8.8 & 26.9 & $<.001$ \\
\hline Waist circumference, $\mathrm{cm}$ & $93.3 \pm 13.5$ & $100.0 \pm 15.7$ & .002 \\
\hline Diabetes mellitus & 5.1 & 4.9 & .97 \\
\hline Relevant alcohol intake ${ }^{b}$ & 10.0 & 11.8 & .65 \\
\hline Cigarette consumption, pack-years & $9.8 \pm 13.7$ & $10.8 \pm 14.9$ & .59 \\
\hline CAT score, points & $16.7 \pm 7.4$ & $17.7 \pm 8.8$ & .36 \\
\hline LTOT & 25.5 & 26.4 & .87 \\
\hline \multicolumn{4}{|l|}{ Liver status } \\
\hline Liver stiffness, $k P a$ & $4.8 \pm 1.1$ & $12.9 \pm 9.4$ & $<.001$ \\
\hline $\mathrm{CAP}, d B / m$ & $261 \pm 54$ & $288 \pm 62$ & $<.001$ \\
\hline $\mathrm{CAP} \geq 280 \mathrm{~dB} / \mathrm{m}$ & 33.9 & 55.0 & .001 \\
\hline APRI, units & $0.32 \pm 0.15$ & $0.68 \pm 0.63$ & $<.001$ \\
\hline APRI $\geq 0.50$ units & 10.8 & 46.2 & $<.001$ \\
\hline APRI $\geq 1.00$ units & 0.3 & 15.4 & $<.001$ \\
\hline HepaScore, units & $0.37 \pm 0.29$ & $0.63 \pm 0.34$ & $<.001$ \\
\hline HepaScore $\geq 0.48$ units & 29.1 & 61.5 & $<.001$ \\
\hline HepaScore $\geq 0.72$ units & 17.6 & 53.8 & $<.001$ \\
\hline ALT, \% of ULN & $72.8 \pm 40.9$ & $103.0 \pm 72.2$ & $<.001$ \\
\hline $\mathrm{ALT} \geq \mathrm{ULN}$ & 13.3 & $3 \overline{7} .9$ & $<.001$ \\
\hline $\mathrm{ALT}, U / L$ & $31.0 \pm 19.4$ & $48.2 \pm 35.0$ & $<.001$ \\
\hline AST, \% of ULN & $68.6 \pm 24.5$ & $92.8 \pm 46.1$ & $<.001$ \\
\hline AST $\geq$ ULN & 7.2 & 30.5 & $<.001$ \\
\hline AST, $U / L$ & $28.5 \pm 10.0$ & $43.2 \pm 22.8$ & $<.001$ \\
\hline GGT, \% of ULN & $73.1 \pm 59.2$ & $185.8 \pm 236.3$ & $<.001$ \\
\hline GGT $\geq$ ULN & 16.2 & 48.4 & $<.001$ \\
\hline GGT, $\bar{U} / L$ & $36.7 \pm 33.5$ & $103.7 \pm 137.4$ & $<.001$ \\
\hline ALP, $\%$ of ULN & $64.2 \pm 20.9$ & $70.3 \pm 28.0$ & .03 \\
\hline ALP $\geq$ ULN & 8.3 & 9.7 & .67 \\
\hline ALP, $\bar{U} / L$ & $73.2 \pm 20.3$ & $85.5 \pm 33.1$ & $<.001$ \\
\hline Bilirubin, \% of ULN & $48.6 \pm 28.8$ & $57.7 \pm 26.9$ & .01 \\
\hline Bilirubin $\geq$ ULN & 5.2 & 7.1 & .53 \\
\hline Bilirubin, $\mu \mathrm{mol} / \mathrm{L}$ & $9.1 \pm 5.4$ & $10.9 \pm 5.1$ & .009 \\
\hline GLDH, $\%$ of ULN & $59.0 \pm 52.6$ & $108.5 \pm 112.1$ & $<.001$ \\
\hline $\mathrm{GLDH} \geq \mathrm{ULN}$ & 9.3 & 39.7 & $<.001$ \\
\hline GLDH, U/L & $4.4 \pm 16.8$ & $7.0 \pm 7.6$ & .23 \\
\hline Platelet count, $G / L$ & $244.1 \pm 65.1$ & $193.3 \pm 58.9$ & $<.001$ \\
\hline Platelets $<150 \mathrm{G} / \mathrm{L}$ & 4.7 & 22.0 & $<.001$ \\
\hline INR, units ${ }^{C}$ & $1.00 \pm 0.08$ & $1.07 \pm 0.10$ & $<.001$ \\
\hline Albumin, $g / L$ & $4.5 \pm 0.5$ & $4.3 \pm 0.5$ & .001 \\
\hline \multicolumn{4}{|l|}{ Lipid metabolism, mg/dL } \\
\hline Triglycerides & $96.6 \pm 44.2$ & $117.5 \pm 70.8$ & .001 \\
\hline Cholesterol & $214.9 \pm 44.9$ & $207.4 \pm 40.4$ & .15 \\
\hline LDL cholesterol & $124.3 \pm 37.0$ & $116.6 \pm 35.6$ & .09 \\
\hline VLDL cholesterol & $9.7 \pm 3.4$ & $9.2 \pm 3.6$ & .49 \\
\hline
\end{tabular}


Supplementary Table 8. Continued

\begin{tabular}{lccc}
\hline \multicolumn{1}{c}{ Variable } & $\begin{array}{c}\mathrm{Pi}^{\star} Z Z, \mathrm{LSM}<7.1 \mathrm{kPa} \\
(\mathrm{n}=308)\end{array}$ & $\begin{array}{c}P i^{\star} Z Z, \text { LSM } \geq 7.1 \mathrm{kPa} \\
\text { (n=95) }\end{array}$ & $\begin{array}{c}P \text { value } \\
\text { (univariable) }\end{array}$ \\
\hline Further blood tests & & $0.94 \pm 0.27$ & .77 \\
Creatinine, $m g / d L$ & $0.97 \pm 0.73$ & $15.4 \pm 1.5$ & .08 \\
Hemoglobin, $m g / d L$ & $15.0 \pm 1.4$ & $7.1 \pm 2.2$ & .70 \\
White blood cell count, $\mathrm{G} / \mathrm{L}$ & $7.2 \pm 2.0$ & $2.8 \pm 3.9$ & .89 \\
C-reactive protein, $m g / d L$ & $2.9 \pm 6.9$ & & \\
\hline
\end{tabular}

NOTE. Quantitative measures are expressed as mean \pm SD or as relative frequency (\%).

ALP, alkaline phosphatase; BMI, body mass index; GLDH, glutamate dehydrogenase; INR, international normalized ratio; LDL, low-density lipoprotein; LLN, sex-specific lower limit of normal; ULN, sex-specific upper limit of normal; VLDL, very-lowdensity lipoprotein.

${ }^{a} A A T$ serum levels of $\mathrm{Pi}^{\star} \mathrm{ZZ}$ subjects without $\mathrm{AAT}$ augmentation therapy are shown. AAT serum levels in all $\mathrm{Pi}{ }^{\star} \mathrm{ZZ}$ subjects were $74.0 \pm 54.3 \mathrm{mg} / \mathrm{dL}(\mathrm{LSM}<7.1 \mathrm{kPa})$ vs $67.7 \pm 47.0 \mathrm{mg} / \mathrm{dL}(\mathrm{LSM} \geq 7.1 \mathrm{kPa}) ; P=.33$.

${ }^{b}$ Alcohol intake $>12 \mathrm{~g} / \mathrm{d}$ for women and $>24 \mathrm{~g} / \mathrm{d}$ for men (individuals with alcohol consumption $>40 \mathrm{~g} / \mathrm{d}$ for females or $>60$ $\mathrm{g} / \mathrm{d}$ for males had been excluded a priori).

${ }^{c}$ Only patients not taking anticoagulant medication were considered.

Supplementary Table 9. Characteristics of Homozygous Carriers of the $\alpha 1$-Antitrypsin $\mathrm{Pi}^{\star} \mathrm{Z}$ Variant $\left(\mathrm{Pi}^{\star} \mathrm{ZZ}\right)$ With and Without an Aspartate Aminotransferase to Platelet Ratio Index Suggesting Significant Liver Fibrosis

\begin{tabular}{|c|c|c|c|}
\hline Variable & $\begin{array}{c}\mathrm{Pi}^{\star} \mathrm{ZZ} \text { carriers, } \\
\text { APRI }<0.50 \text { units } \\
(\mathrm{n}=312)\end{array}$ & $\begin{array}{c}\mathrm{Pi}^{\star} \mathrm{ZZ} \text { carriers, } \\
\text { APRI } \geq 0.50 \text { units } \\
(\mathrm{n}=74)\end{array}$ & $\begin{array}{c}P \text { value } \\
\text { (univariable) }\end{array}$ \\
\hline \multicolumn{4}{|l|}{ Characteristics } \\
\hline Age, $y$ & $53.6 \pm 13.4$ & $56.4 \pm 11.9$ & .11 \\
\hline Women & 52.2 & 16.2 & $<.001$ \\
\hline $\mathrm{BMI}, \mathrm{kg} / \mathrm{m}^{2}$ & $24.5 \pm 4.3$ & $26.0 \pm 4.6$ & .009 \\
\hline Diabetes mellitus & 4.6 & 6.1 & .63 \\
\hline CAT score, points & $16.9 \pm 7.7$ & $17.4 \pm 8.1$ & .61 \\
\hline LTOT & 24.3 & 31.9 & .19 \\
\hline \multicolumn{4}{|l|}{ Liver status } \\
\hline Liver stiffness, $\mathrm{kPa}$ & $5.7 \pm 3.0$ & $11.0 \pm 10.5$ & $<.001$ \\
\hline $\mathrm{CAP}, d B / m$ & $262.1 \pm 54.9$ & $289.0 \pm 61.1$ & .001 \\
\hline GGT, \% of ULN & $76.0 \pm 60.9$ & $207.0 \pm 262.6$ & $<.001$ \\
\hline ALP, $\%$ of ULN & $74.6 \pm 20.3$ & $81.7 \pm 36.2$ & .53 \\
\hline Bilirubin, \% of ULN & $49.7 \pm 29.1$ & $55.8 \pm 27.7$ & .11 \\
\hline GLDH, \% of ULN & $54.1 \pm 37.4$ & $131.0 \pm 126.6$ & $<.001$ \\
\hline Platelet count, $G / L$ & $247.6 \pm 61.3$ & $165.8 \pm 47.0$ & $<.001$ \\
\hline Triglycerides, $\mathrm{mg} / \mathrm{dL}$ & $97.2 \pm 45.7$ & $119.6 \pm 71.8$ & .001 \\
\hline
\end{tabular}

NOTE. Quantitative measures are expressed as mean \pm SD or as relative frequency (\%).

ALP, alkaline phosphatase; BMI, body mass index; GLDH, glutamate dehydrogenase; ULN, sex-specific upper limit of normal. ${ }^{a}$ Only patients not taking anticoagulant medication were considered. 
Supplementary Table 10. Characteristics of Homozygous Carriers of the $\alpha 1$-Antitrypsin $\mathrm{Pi}^{\star} \mathrm{Z}$ Variant ( $\left.\mathrm{Pi}^{\star} \mathrm{ZZ}\right)$ With and Without a HepaScore Suggesting Significant Liver Fibrosis

\begin{tabular}{|c|c|c|c|}
\hline Characteristic & $\begin{array}{c}\mathrm{Pi}^{\star} \mathrm{ZZ} \text { carriers, } \\
\text { HepaScore }<0.48 \text { units } \\
(\mathrm{n}=149)\end{array}$ & $\begin{array}{c}\mathrm{Pi}^{\star} \mathrm{ZZ} \text { carriers, } \\
\text { HepaScore } \geq 0.48 \text { units } \\
(\mathrm{n}=85)\end{array}$ & $\begin{array}{c}P \text { value } \\
\text { (univariable) }\end{array}$ \\
\hline \multicolumn{4}{|l|}{ Characteristics } \\
\hline Age, $y$ & $52.0 \pm 11.2$ & $61.2 \pm 11.4$ & $<.001$ \\
\hline Women & 59.1 & 25.9 & $<.001$ \\
\hline $\mathrm{BMI}, \mathrm{kg} / \mathrm{m}^{2}$ & $24.9 \pm 4.3$ & $25.1 \pm 4.7$ & .66 \\
\hline $\mathrm{BMI} \geq 30 \mathrm{~kg} / \mathrm{m}^{2}$ & 14.1 & 17.6 & .47 \\
\hline Waist circumference, $\mathrm{cm}$ & $94.2 \pm 12.3$ & $97.8 \pm 15.1$ & .07 \\
\hline Diabetes mellitus & 5.4 & 7.1 & .56 \\
\hline CAT score, points & $16.2 \pm 8.1$ & $18.0 \pm 7.4$ & .09 \\
\hline LTOT & 26.8 & 34.1 & .24 \\
\hline \multicolumn{4}{|l|}{ Liver status } \\
\hline Liver stiffness, $\mathrm{kPa}$ & $5.5 \pm 3.4$ & $8.6 \pm 8.6$ & $<.001$ \\
\hline $\mathrm{CAP}, d B / m$ & $266.8 \pm 55.0$ & $277.6 \pm 63.0$ & .19 \\
\hline APRI, units & $0.21 \pm 0.11$ & $0.81 \pm 0.17$ & $<.001$ \\
\hline \multicolumn{4}{|l|}{ Biochemistry } \\
\hline ALT, \% of ULN & $81.0 \pm 50.1$ & $89.6 \pm 41.1$ & .18 \\
\hline AST, \% of ULN & $72.6 \pm 27.1$ & $83.2 \pm 30.0$ & .006 \\
\hline GGT, $\%$ of ULN & $75.0 \pm 48.1$ & $109.6 \pm 115.8$ & .002 \\
\hline ALP, \% of ULN & $74.1 \pm 20.9$ & $83.0 \pm 29.1$ & .01 \\
\hline Bilirubin, \% of ULN & $45.1 \pm 20.8$ & $53.4 \pm 29.6$ & .01 \\
\hline GLDH, \% of ULN & $60.3 \pm 65.0$ & $80.7 \pm 64.5$ & .02 \\
\hline Platelet count, $G / L$ & $247.3 \pm 60.8$ & $211.4 \pm 64.0$ & $<.001$ \\
\hline Triglycerides, $m g / d L$ & $90.2 \pm 36.0$ & $112.1 \pm 58.3$ & .001 \\
\hline
\end{tabular}

NOTE. Quantitative measures are expressed as mean \pm SD or as relative frequency (\%).

ALP, alkaline phosphatase; BMI, body mass index; GLDH, glutamate dehydrogenase; ULN, sex-specific upper limit of normal. ${ }^{a}$ Only patients not taking anticoagulant medication were considered. 